Preconditioning steady-state Navier-Stokes equations with random data

Powell, Catherine E. and Silvester, David J. 2012

MIMS EPrint: 2012.35

Manchester Institute for Mathematical Sciences

School of Mathematics

The University of Manchester

\footnotetext{
Reports available from: http://eprints.maths.manchester.ac.uk/

And by contacting: The MIMS Secretary

School of Mathematics

The University of Manchester

Manchester, M13 9PL, UK
} 


\title{
PRECONDITIONING STEADY-STATE NAVIER-STOKES EQUATIONS WITH RANDOM DATA
}

\author{
CATHERINE E. POWELL* AND DAVID J. SILVESTER*
}

\begin{abstract}
We consider the numerical solution of the steady-state Navier-Stokes equations with uncertain data. Specifically, we treat the case of uncertain viscosity, which results in a flow with an uncertain Reynolds number. After linearization, we apply a stochastic Galerkin finite element method, combining standard inf-sup stable Taylor-Hood approximation on the spatial domain (on highly stretched grids), with orthogonal polynomials in the stochastic parameter. This yields a sequence of non-symmetric saddle-point problems with Kronecker product structure. The novel contribution of this study lies in the construction of efficient block triangular preconditioners for these discrete systems, for use with GMRES. Crucially, the preconditioners are robust with respect to the discretization and statistical parameters, and we exploit existing deterministic solvers based on the so-called Pressure Convection-Diffusion and Least-Squares Commutator approximations.
\end{abstract}

Keywords Navier-Stokes equations, random data, stochastic Galerkin method, finite elements, mixed approximation, preconditioning, multigrid, uncertainty quantification.

AMS subject classifications. 35R60, 65C20,65F10, 65N22, 65N30

1. Introduction. Our starting point is the steady-state Navier-Stokes equations for an incompressible fluid with a fixed viscosity $\nu>0$. The fluid moves inside a domain $\Omega \subset \mathbb{R}^{n}$ and the velocity is nonzero on some part of the boundary $\Gamma$. We want to compute the fluid velocity $\vec{u}: \Omega \rightarrow \mathbb{R}^{n}$ and the pressure $p: \Omega \rightarrow \mathbb{R}$ satisfying

$$
\begin{aligned}
-\nu \nabla^{2} \vec{u}+\vec{u} \cdot \nabla \vec{u}+\nabla p & =\overrightarrow{0} & & \text { in } \Omega, \\
\nabla \cdot \vec{u} & =0 & & \text { in } \Omega .
\end{aligned}
$$

For ease of exposition we only consider two-dimensional spatial domains $\Omega \subset \mathbb{R}^{2}$. We also focus exclusively on inflow-outflow configurations, where the boundary comprises two non-overlapping segments $\Gamma_{D} \cup \Gamma_{N}$ associated with a specified inlet flow field $\vec{g}$ (set to zero at a fixed wall) and a standard natural outflow boundary condition,

$$
\begin{aligned}
\vec{u} & =\vec{g} & & \text { on } \Gamma_{D}, \\
\nu \nabla \vec{u} \cdot \vec{n}-p \vec{n} & =\overrightarrow{0} & & \text { on } \Gamma_{N} .
\end{aligned}
$$

We assume that $\left|\Gamma_{N}\right| \neq 0$ to ensure the pressure $p$ is uniquely specified by the outflow condition (1.4). In the case of fully-developed parallel flow, $\vec{u} \cdot \vec{t}=0$ and the pressure at the outflow boundary will automatically be set to zero (see Elman et al. [4, p. 216]).

A simple approach to solving systems of nonlinear partial differential equations is to apply a linearization scheme, followed by a mixed finite element method, and then solve the resulting sequence of discrete systems using appropriate preconditioned Krylov subspace methods. Before progress can be made, however, all the input data needs to be supplied. For the Navier-Stokes equations (1.1)-(1.2), we require the viscosity $\nu$; the spatial geometry, i.e., the domain $\Omega$, and the boundary data $\vec{g}$ (which depends on the configuration of the boundary $\Gamma_{D}$ ). In real-life applications, where we rarely have access to all of this information, hypothetical data is often used. An alternative modelling approach is to represent unknown inputs as random variables and view the solution variables, also, as random variables. Numerical methods are then required to perform uncertainty quantification, the process of determining statistical information about the solution, given a statistical description of the input data.

* School of Mathematics, University of Manchester, Oxford Road, Manchester, M13 9PL, United Kingdom. E-mail: catherine.powell@manchester.ac.uk; david.silvester@manchester.ac.uk 
1.1. Outline. In Section 2, we review the numerical solution of the deterministic steady-state Navier-Stokes equations (1.1)-(1.4) via stable mixed finite element methods, focusing on the classical flow over a backward-facing step problem. We combine Picard iteration with finite elements on stretched grids and derive a sequence of structured saddle-point systems. State of the art preconditioners based on the Pressure Convection-Diffusion (PCD) and Least-Squares Commutator (LSC) approximations are also reviewed. In Section 3, and in the sequel, we consider a more challenging modelling situation where the viscosity in the flow equations is a random variable. We show that this is equivalent to a scenario where the volume of fluid moving into the channel is uncertain. In Section 4, we derive a fully discrete problem by combining Picard iteration with a stochastic Galerkin mixed finite element method. Finally, in Sections 5 and 6, we demonstrate that the deterministic PCD and LSC preconditioners can be used as building blocks to provide new, effective solvers for the saddle-point systems arising in the nonlinear iteration for the stochastic Navier-Stokes problem.

2. Deterministic Navier-Stokes problem. The flow inside a channel with a sudden expansion, or flow over a backward-facing step, is a classical test problem. The geometry is shown in Figure 2.1. The re-entrant corner is positioned at the origin $(0,0)$ and we fix $d=1$ and $L=5$. A quadratic flow profile, $\vec{g}=\left(g_{x}, g_{y}\right)=\left(4 y(d-y) / d^{3}, 0\right)$, is imposed on the inflow boundary, $\Gamma_{\text {in }}$, and a steady no-flow condition is applied on $\Gamma_{\text {wall }}$. At the outflow boundary, $\Gamma_{\text {out }}$, the natural condition (1.4) forces the mean outflow pressure to be zero. To non-dimensionalize the Navier-Stokes equations, we define the reference length $\bar{L}$ as the length of the outflow channel and the reference velocity $\bar{U}$ as the average inlet velocity, $V:=\left(\frac{1}{d}\right) \int_{0}^{d} g_{x} d y$ (see Gresho et al. [8]). If $d=1$, then $\bar{U}$ is the volume of fluid flowing into the channel. With the configuration shown in Figure 2.1 and $d=1$, we have $\bar{L}=2, \bar{U}=2 / 3$ and the Reynolds number is

$$
\operatorname{Re}:=\frac{\bar{L} \bar{U}}{\nu}=\frac{4}{3 \nu} \text {. }
$$

This gives a measure of the relative contributions of the diffusion and convection terms in (1.1) and is an important parameter in testing solver robustness.

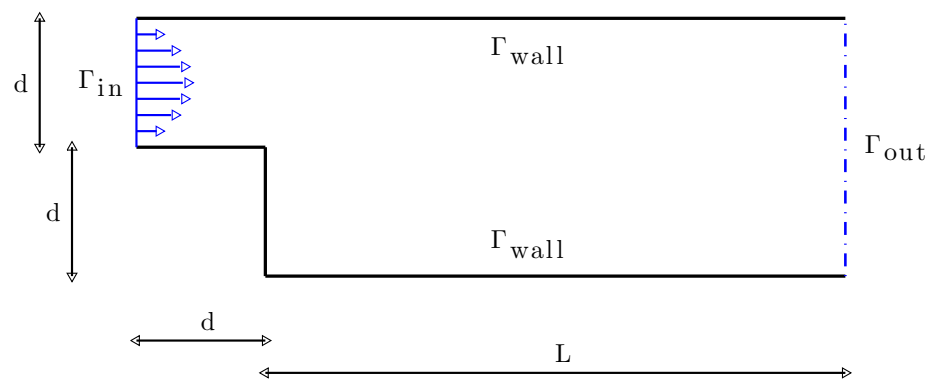

FIG. 2.1. The backward-facing step domain.

Spatial discretization is accomplished herein via stable, conforming, mixed finite elements (see e.g. Elman et al. [4, Chapter 5]). Specifically, we apply $Q_{2}-Q_{1}$ (biquadratic velocity, bilinear continuous pressure) approximation. The singularity in the solution at the re-entrant corner is a very important feature of the test problem and spatial discretization needs to be done carefully. We use non-uniform grids of rectangles, as shown in Figure 2.2. Observe that there are highly stretched elements in both coordinate directions along the lines $x=0$ and $y=0$. Such grids are known to provide a stiff test for solver strategies that employ algebraic multigrid (AMG). If we set the viscosity to be $\nu=1 / 50$ then $\operatorname{Re}=200 / 3$, and a recirculating eddy develops in the flow, downstream of the step. A sample finite element solution computed on a stretched grid of 1,536 $Q_{2}-Q_{1}$ elements is shown in Figure 2.3. 


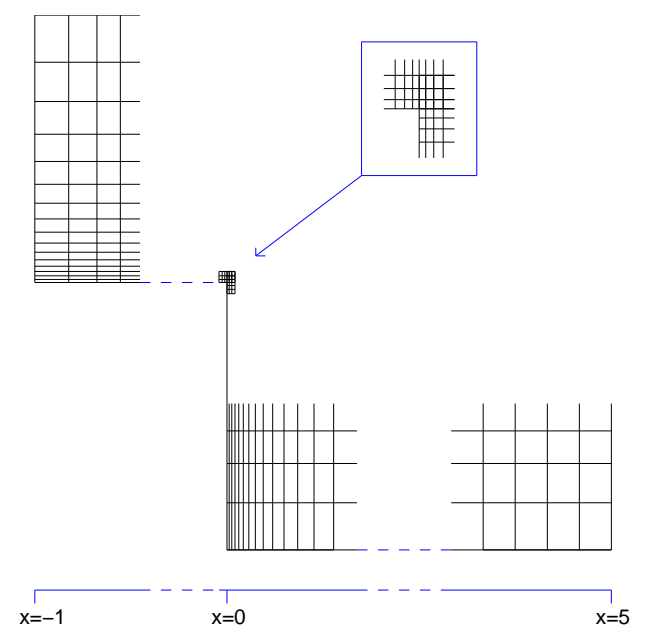

FIG. 2.2. Grid details (plotted to scale) showing the refinement near the corner $(\times 3$ in the zoom).
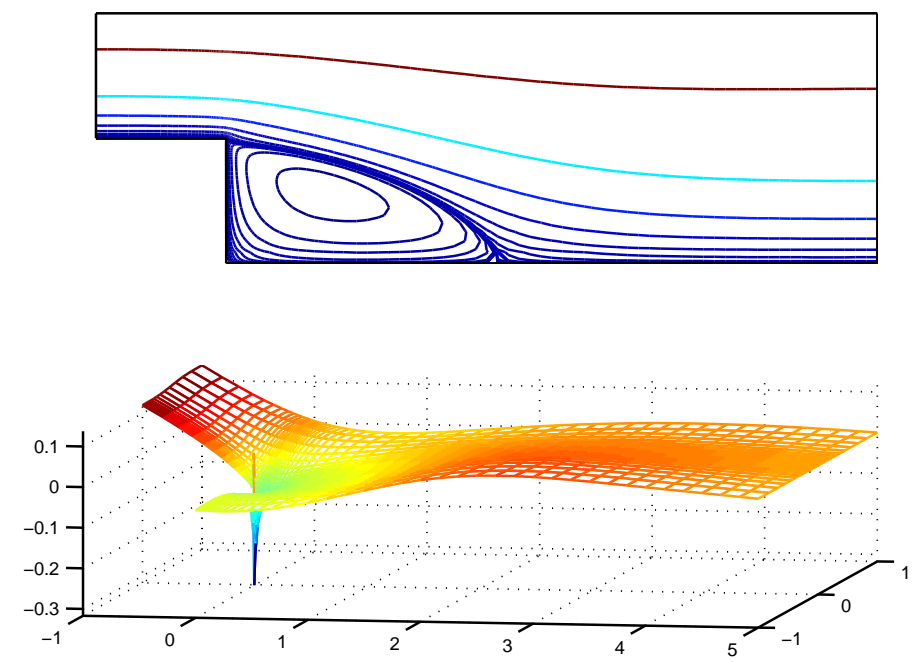

FiG. 2.3. Streamlines of finite element flow field (top) and pressure field (bottom) for the backwardfacing step test problem with $\nu=1 / 50$ and $\mathrm{Re}=200 / 3$. The locally refined grid of 1,536 elements yields $n_{p}=1,625$ and $n_{u}=6,321$, a total of $2 n_{u}+n_{p}=14,267$ degrees of freedom.

2.1. Discrete problem. As usual, the grid subdivision is denoted by $\mathscr{T}_{h}$ and we associate the parameter $h$ with the length of the longest edge. Denoting the velocity finite element solution space by $\boldsymbol{X}_{E}^{h} \subset \boldsymbol{H}_{E}^{1}(\Omega)$ (interpolating $\vec{g}$ on the inflow boundary $\left.\Gamma_{D}\right)$ and the pressure space by $M^{h} \subset L^{2}(\Omega)$, the fully discrete problem is: compute $\vec{u}_{h} \in \boldsymbol{X}_{E}^{h}$ and $p_{h} \in M^{h}$ satisfying the Galerkin formulation,

$$
\begin{aligned}
\nu\left(\nabla \vec{u}_{h}, \nabla \vec{v}_{h}\right)+\left(\vec{u}_{h} \cdot \nabla \vec{u}_{h}, \vec{v}_{h}\right)-\left(p_{h}, \nabla \cdot \vec{v}_{h}\right) & =0, \\
\left(\nabla \cdot \vec{u}_{h}, q_{h}\right) & =0,
\end{aligned}
$$

for all test functions $\vec{v}_{h} \in \boldsymbol{X}_{0}^{h}$ (incorporating zero boundary conditions on $\Gamma_{D}$ ) and $q_{h} \in M^{h}$. Herein, $(\cdot, \cdot)$ represents the $L^{2}(\Omega)$ inner product. Given initial guesses $\vec{u}_{h}^{0}$ and $p_{h}^{0}$, lagging the convection coefficient leads to simple Picard iteration for a sequence 
$\left(\vec{u}_{h}^{n}, p_{h}^{n}\right) \in \boldsymbol{X}_{E}^{h} \times M_{h}, n=1,2, \ldots$, of finite element solutions satisfying

$$
\begin{aligned}
\nu\left(\nabla \vec{u}_{h}^{n+1}, \nabla \vec{v}_{h}\right)+\left(\vec{u}_{h}^{n} \cdot \nabla \vec{u}_{h}^{n+1}, \vec{v}_{h}\right)-\left(p_{h}^{n+1}, \nabla \cdot \vec{v}_{h}\right) & =0, \\
\left(\nabla \cdot \vec{u}_{h}^{n+1}, q_{h}\right) & =0,
\end{aligned}
$$

for all $\vec{v}_{h} \in \boldsymbol{X}_{0}^{h}$ and $q_{h} \in M^{h}$.

To see the structure of the sequence of linear algebra problems associated with (2.3)-(2.4), we need specific basis sets for the approximation spaces:

$$
\boldsymbol{X}_{0}^{h}=\operatorname{span}\left\{\left[\begin{array}{c}
\phi_{i} \\
0
\end{array}\right],\left[\begin{array}{c}
0 \\
\phi_{i}
\end{array}\right]\right\}_{i=1}^{n_{u}}, \quad M^{h}=\operatorname{span}\left\{\psi_{j}\right\}_{j=1}^{n_{p}} .
$$

We compute the discrete updates $\vec{d}_{h}^{n}$ and $\delta_{h}^{n}$ so that the solution at step $n+1$ is

$$
\vec{u}_{h}^{n+1}=\vec{u}_{h}^{n}+\vec{d}_{h}^{n}, \quad p_{h}^{n+1}=p_{h}^{n}+\delta_{h}^{n} .
$$

Then, given the expansions

$$
\vec{d}_{h}^{n}=\left[\begin{array}{c}
\sum_{i=1}^{n_{u}} \alpha_{i}^{x, n} \phi_{i} \\
\sum_{i=1}^{n_{u}} \alpha_{i}^{y, n} \phi_{i}
\end{array}\right], \quad \delta_{h}^{n}=\sum_{j=1}^{n_{p}} \alpha_{j}^{p, n} \psi_{j},
$$

the vectors $\boldsymbol{\alpha}^{u, n}=\left[\boldsymbol{\alpha}^{x, n}, \boldsymbol{\alpha}^{y, n}\right], \boldsymbol{\alpha}^{p, n}$ are found by solving the saddle-point system:

$$
\left(\begin{array}{cc}
\boldsymbol{F}_{\nu}^{n} & B^{T} \\
B & 0
\end{array}\right)\left(\begin{array}{l}
\boldsymbol{\alpha}^{u, n} \\
\boldsymbol{\alpha}^{p, n}
\end{array}\right)=\left(\begin{array}{l}
\boldsymbol{f}^{u, n} \\
\boldsymbol{f}^{p, n}
\end{array}\right) .
$$

The vectors on the right-hand side of (2.6) are residuals associated with the updates and $B=\left[B_{x}, B_{y}\right]$ is the discrete divergence operator with components

$$
B_{x}:=\left[B_{x}\right]_{j i}=-\left(\psi_{j}, \frac{\partial \phi_{i}}{\partial x}\right), \quad B_{y}:=\left[B_{y}\right]_{j i}=-\left(\psi_{j}, \frac{\partial \phi_{i}}{\partial y}\right),
$$

for $j=1, \ldots, n_{p}$ and $i=1, \ldots, n_{u} . \boldsymbol{F}_{\nu}^{n}$ is the discrete convection-diffusion operator:

$$
\boldsymbol{F}_{\nu}^{n}:=\nu \boldsymbol{A}+\boldsymbol{N}\left(\vec{u}_{h}^{n}\right)
$$

where $\boldsymbol{A}$ and $\boldsymbol{N}$ are block-diagonal matrices with (scalar) components,

$$
\begin{aligned}
A & :=[A]_{i j}=\left(\nabla \phi_{i}, \nabla \phi_{j}\right), \quad i, j=1, \ldots, n_{u}, \\
N\left(\vec{u}_{h}\right) & :=[N]_{i j}=\left(\vec{u}_{h} \cdot \nabla \phi_{i}, \phi_{j}\right) \quad i, j=1, \ldots, n_{u} .
\end{aligned}
$$

Note that the inflow-outflow boundary condition ensures that $B^{T}$ has full rank and so the system in (2.6) is nonsingular.

2.2. Preconditioned GMRES. Our preferred solver is right-preconditioned GMRES with a preconditioner that is specially tailored to the matrix in (2.6). First, we express (2.6) (omitting the subscripts/superscripts) with a preconditioner $\mathscr{P}$ so that

$$
\left(\begin{array}{cc}
\boldsymbol{F} & B^{T} \\
B & 0
\end{array}\right) \mathscr{P}^{-1} \quad \mathscr{P}\left(\begin{array}{c}
\boldsymbol{\alpha}^{u} \\
\boldsymbol{\alpha}^{p}
\end{array}\right)=\left(\begin{array}{c}
\boldsymbol{f}^{u} \\
\boldsymbol{f}^{p}
\end{array}\right)
$$

The ideal block-triangular preconditioner

$$
\mathscr{P}:=\left(\begin{array}{cc}
\boldsymbol{F} & B^{T} \\
0 & -S
\end{array}\right) \approx\left(\begin{array}{cc}
\boldsymbol{F} & B^{T} \\
B & 0
\end{array}\right)
$$

where $S$ is the Schur complement $S:=B \boldsymbol{F}^{-1} B^{T}$, is motivated by the identity

$$
\left(\begin{array}{cc}
\boldsymbol{F} & B^{T} \\
B & 0
\end{array}\right) \underbrace{\left(\begin{array}{cc}
\boldsymbol{F}^{-1} & \boldsymbol{F}^{-1} B^{T} S^{-1} \\
0 & -S^{-1}
\end{array}\right)}_{\mathscr{P}^{-1}} \equiv\left(\begin{array}{cc}
I & 0 \\
B \boldsymbol{F}^{-1} & I
\end{array}\right) .
$$


This shows that the eigenvalues of the preconditioned matrix are clustered at unity. The matrix on the right-hand side has Jordan blocks of dimension two, which implies that right-preconditioned GMRES converges in two iterations, independently of the convection coefficient $\vec{u}_{h}^{n}$ and the specific values of $h$ and $\nu$. Observe that the action of $\mathscr{P}^{-1}$ can be applied as a three-step process. First, we solve systems associated with the Schur complement $S$, second, we perform a matrix-vector multiplication with $B^{T}$, and finally we solve two scalar systems associated with the convection-diffusion matrix $\boldsymbol{F}$.

It is not practical to work with the Schur complement $S$ and there are two state of the art strategies that circumvent its use (e.g. see [4]). The first approach, referred to as Pressure Convection-Diffusion (PCD) preconditioning, is a triple product approximation. The ingredients are a matrix-vector multiplication with a matrix $F_{p}$ (obtained by constructing $\boldsymbol{F}=\boldsymbol{F}_{\nu}^{n}$ in (2.8) with velocity basis functions $\phi_{i}$ replaced by pressure basis functions $\psi_{j}$; see Section 5.2), together with linear solves for a pressure diffusion matrix $A_{p}$ and a pressure mass matrix $Q_{p}$. That is,

$$
S^{-1}=\left(B \boldsymbol{F}^{-1} B^{T}\right)^{-1} \approx Q_{p}^{-1} F_{p} A_{p}^{-1} .
$$

Elman \& Tuminaro [5] suggest implementing PCD preconditioning via

$$
S^{-1}=\left(B \boldsymbol{F}^{-1} B^{T}\right)^{-1} \approx Q_{*}^{-1} F_{p} A_{*}^{-1},
$$

where $Q_{*}$ is the diagonal of $Q_{p}$ and $A_{*}:=B \boldsymbol{M}_{*}^{-1} B^{T}$, where $\boldsymbol{M}_{*}$ is the diagonal of the velocity mass matrix. The second approach, referred to as Least-Squares Commutator (LSC) preconditioning, avoids the construction of $F_{p}$ and is given by

$$
S^{-1}=\left(B \boldsymbol{F}^{-1} B^{T}\right)^{-1} \approx A_{*}^{-1}\left(B \boldsymbol{M}_{*}^{-1} \boldsymbol{F} \boldsymbol{M}_{*}^{-1} B^{T}\right) A_{*}^{-1} .
$$

The action of $A_{*}^{-1}$ in (2.14) and (2.15) can also be applied inexactly using AMG (typically with one or two V-cycles). Studies of PCD and LSC preconditioning for (1.1)-(1.2) are mature and a summary of their performance for the deterministic backward-facing step problem is presented in [4, Section 8.2]. We focus, now, on a more realistic modelling situation where there is uncertainty in the input data.

3. Navier-Stokes problems with uncertain data. Suppose that the fluid viscosity $\nu$ is spatially constant but that its value is not known precisely. Instead of guessing a value, we can model $\nu$ as a random variable on a probability space $(\Xi, F, \mathbb{P})$. Here, $\Xi$ denotes the set of outcomes, $F$ is a $\sigma$-algebra of events and $\mathbb{P}: F \rightarrow[0,1]$ is a probability measure. The corresponding Navier-Stokes velocity and pressure are also random variables and the numerical solution of the flow equations is far more challenging.

Given a (deterministic) vector function $\vec{g}=\vec{g}(\vec{x})$ on $\Gamma_{D}$, the boundary value problem for the Navier-Stokes equations with a random viscosity reads as follows: find $\vec{u}=$ $\vec{u}(\vec{x}, \omega)$ and $p=p(\vec{x}, \omega)$ such that $\mathbb{P}$-a.s (i.e., with probability one),

$$
\begin{aligned}
-\nu(\omega) \nabla^{2} \vec{u}+\vec{u} \cdot \nabla \vec{u}+\nabla p & =\overrightarrow{0} & & \text { in } \Omega, \\
\nabla \cdot \vec{u} & =0 & & \text { in } \Omega, \\
\vec{u} & =\vec{g} & & \text { on } \Gamma_{D}, \\
\nu \nabla \vec{u} \cdot \vec{n}-p \vec{n} & =\overrightarrow{0} & & \text { on } \Gamma_{N} .
\end{aligned}
$$

The solution variables $\vec{u}, p: \Omega \times \Xi \rightarrow \mathbb{R}$ are now random fields. One way to interpret this is that realizations of $\nu$ give rise to deterministic functions $\vec{u}(\cdot, \omega), p(\cdot, \omega)$ on $\Omega$ which satisfy the standard deterministic Navier-Stokes equations.

Why would we want to solve the Navier-Stokes equations with a random viscosity? The uncertainty could physically stem from measurement error in $\nu$. Note, however, that if $\nu$ is a random variable then so is the Reynolds number, Re. Indeed, for the fixed spatial geometry in Figure 2.1, we have,

$$
\operatorname{Re}(\omega):=\frac{\bar{L} \bar{U}}{\nu(\omega)}=\frac{2 d \cdot 2}{\nu(\omega) \cdot 3 d}=\frac{4}{3 \nu(\omega)} .
$$


Now suppose we want to investigate the properties of the flow in the presence of an uncertain Reynolds number $\operatorname{Re}(\omega)$, with a particular statistical distribution. One way to achieve this is by fixing the geometry and the boundary conditions and choosing $\nu$ to be an appropriate random variable, but there are other possibilities.

Consider the rescaled fields $\vec{U}(\vec{x}, \omega):=\vec{u}(\vec{x}, \omega) / \nu(\omega)$ and $P(\vec{x}, \omega):=p(\vec{x}, \omega) / \nu^{2}(\omega)$ where $\vec{u}$ and $p$ satisfy (3.1)-(3.4). Then, $\mathbb{P}$-a.s, $\vec{U}(\vec{x}, \omega), P(\vec{x}, \omega)$ satisfy

$$
\begin{aligned}
-\nabla^{2} \vec{U}+\vec{U} \cdot \nabla \vec{U}+\nabla P & =\overrightarrow{0} & & \text { in } \Omega, \\
\nabla \cdot \vec{U} & =0 & & \text { in } \Omega, \\
\vec{U} & =\vec{G} & & \text { on } \Gamma_{D}, \\
\nabla \vec{U} \cdot \vec{n}-P \vec{n} & =\overrightarrow{0} & & \text { on } \Gamma_{N},
\end{aligned}
$$

where $\vec{G}=\vec{G}(\vec{x}, \omega):=\vec{g}(\vec{x}) / \nu(\omega)$. The boundary value problem (3.5)-(3.8) consists of the steady-state Navier-Stokes equations with unit viscosity, subject to the homogeneous Neumann boundary condition (3.8) and the stochastic Dirichlet boundary condition (3.7). For the inflow-outflow configuration and spatial geometry shown in Figure 2.1, with $d=1$, the inlet profile $\vec{g}(\vec{x})$ is now scaled by $1 / \nu(\omega)$. Physically, this means that the (quadratic) shape of the inflow profile is fixed, but the maximum velocity is unknown and hence so is the volume $V(\omega):=\int_{0}^{1} g_{x}(y) / \nu(\omega) d y$ of flow moving into the channel. The Reynolds number, $\operatorname{Re}(\omega)=4 /(3 \nu(\omega))$, is unchanged and hence so are the dynamical properties of the flow. If the physical source of uncertainty is the volume of fluid moving into the channel, we have a choice of two problem formulations.

For the backward-facing step problem, there is actually a third possibility. If we fix the volume of fluid moving into the channel to be $\bar{U}=1$ and choose the length of the outflow channel to be $\bar{L}(\omega)=2 d(\omega)$, where the length of the inlet is the random variable $d(\omega)=\nu(\omega)^{-1}$, then it is possible to recover the same Reynolds number distribution by solving a problem with a deterministic viscosity parameter. In this formulation, however, the flow equations need to be solved on a random spatial domain, $\Omega(\omega)$.

3.1. Uncertain viscosity. We focus on the random viscosity formulation (3.1)(3.4). There are two reasons for this: first, the formulation (3.1)-(3.4) is more compatible with our existing software (IFISS, see [2], [13]) and allows maximal reuse of deterministic solvers; second, we can make a direct comparison with results in the literature, see, e.g. Le Maître \& Knio [10, Chapter 6]. Specifically, we consider

$$
\nu(\omega):=\nu_{0}+\nu_{1} \xi(\omega)
$$

where $\nu_{0}, \nu_{1} \in \mathbb{R}^{+}$and $\xi$ is a random variable on $(\Xi, F, \mathbb{P})$. If we know a range of possible values for $\nu$, it is natural to focus on the uniform distribution. With $\xi \sim U(-\sqrt{3}, \sqrt{3})$, we have $\nu \sim U\left(\nu_{\min }, \nu_{\max }\right)$ where

$$
\nu_{\min }:=\nu_{0}-\sqrt{3} \nu_{1}, \quad \nu_{\max }:=\nu_{0}+\sqrt{3} \nu_{1} .
$$

The expectation and variance of the viscosity are then given by

$$
\mathbb{E}[\nu]=\nu_{0}, \quad \operatorname{Var}[\nu]=\nu_{1}^{2},
$$

and we can model different statistical scenarios by varying $\nu_{0}$ and $\nu_{1}$. For a well-posed problem we require that $\mathbb{P}(\nu(\omega)>0)=\mathbb{P}(\{\omega \in \Xi \mid \nu(\omega)>0\})=1$ and so, for a fixed mean viscosity, the choice of standard deviation is constrained by the condition,

$$
\nu_{\text {min }}>0 \Longrightarrow \nu_{1}<\nu_{0} / \sqrt{3} .
$$

For a sufficiently small $\nu_{1}$, if we fix $\nu_{0}=1 / 50$ and select the same finite element grid, we anticipate close agreement between the mean flow and the mean pressure and 
the deterministic solutions shown in Figure 2.3. Recall, when $\nu=1 / 50$, the Reynolds number for the deterministic test problem is $\operatorname{Re}=200 / 3$. For the stochastic problem,

$$
\mathbb{E}[\operatorname{Re}]=\frac{4}{3} \mathbb{E}\left[\nu^{-1}\right]=\frac{2}{3 \sqrt{3} \nu_{1}} \log \left(\frac{\nu_{0}+\sqrt{3} \nu_{1}}{\nu_{0}-\sqrt{3} \nu_{1}}\right)=\frac{2}{3 \sqrt{3} \nu_{1}} \log \left(\frac{\nu_{\max }}{\nu_{\min }}\right)
$$

and when $\nu_{0}=1 / 50$, the expected Reynolds number is close to 200/3 (see Table 3.1).

\begin{tabular}{l|rrrr}
\hline & $\nu_{1}=\nu_{0} / 10$ & $\nu_{1}=2 \nu_{0} / 10$ & $\nu_{1}=3 \nu_{0} / 10$ & $\nu_{1}=4 \nu_{0} / 10$ \\
\hline$\nu_{0}=1 / 50$ & 67.35 & 69.54 & 73.88 & 82.11 \\
$\nu_{0}=1 / 100$ & 134.69 & 139.08 & 147.75 & 164.23 \\
$\nu_{0}=1 / 200$ & 269.38 & 278.17 & 295.51 & 328.46 \\
\hline
\end{tabular}

TABLE 3.1

Expected Reynolds number $\mathbb{E}[\mathrm{Re}]$ for fixed mean viscosity $\nu_{0}=\mathbb{E}[\nu]$ and varying $\nu_{1}$.

3.2. Stokes problem with random viscosity. Before tackling the Navier-Stokes equations, it is instructive to briefly consider the boundary value problem for the linear Stokes equations that correspond to taking the limit $\mathrm{Re} \rightarrow 0$. If the viscosity is a random variable, the problem reads as follows: find $\vec{u}=\vec{u}(\vec{x}, \omega)$ and $p=p(\vec{x}, \omega)$ such that $\mathbb{P}$-a.s,

$$
\begin{aligned}
-\nu(\omega) \nabla^{2} \vec{u}+\nabla p & =\overrightarrow{0} & & \text { in } \Omega, \\
\nabla \cdot \vec{u} & =0 & & \text { in } \Omega, \\
\vec{u} & =\vec{g} & & \text { on } \Gamma_{D}, \\
\nu \nabla \vec{u} \cdot \vec{n}-p \vec{n} & =\overrightarrow{0} & & \text { on } \Gamma_{N} .
\end{aligned}
$$

Rescaling by $\nu^{-1}$ we find that the velocity $\vec{u}$ does not depend on $\nu$, and thus is a deterministic function. We also see that $p(\vec{x}, \omega)=\nu(\omega) p_{\text {det }}(\vec{x})$, where $p_{\text {det }}(\vec{x})$ is the solution to the deterministic Stokes problem with unit viscosity. Thus, for the choice (3.9), the Stokes pressure $p(\vec{x}, \omega)$ depends linearly on the random variable $\xi(\omega)$.

The nonlinear convection term in (3.1) makes it more difficult to characterize the dependence on $\xi(\omega)$ of the Navier-Stokes velocity and pressure. When $\operatorname{Re}=O(1)$, the deterministic Navier-Stokes solution is close to the deterministic Stokes solution. Hence, when realisations of $\operatorname{Re}(\omega)$ are close to order one (e.g., with $\nu_{0}=1$ and $\nu_{1}=0.1$ ), we anticipate a close-to-linear dependence on $\xi(\omega)$ for the pressure solution of the stochastic problem. As the Reynolds number is increased however, we anticipate that nonlinear effects will become increasingly important — realizations of the stochastic Navier-Stokes velocity solution could vary significantly from the flow solution obtained by solving the deterministic Navier-Stokes problem with the Reynolds number set to $\mathbb{E}[\operatorname{Re}]$.

4. A fully discrete problem. To obtain a fully discrete version of (3.1)-(3.4) with $\nu(\omega)$ as in (3.9), we linearize the equations, reformulate as an equivalent parametric problem and then apply a stochastic Galerkin mixed finite element method. The problem nonlinearity is not our focus herein and we take a simple Picard linearization of the convection term (as in Section 2). Hence, at the $n$th iteration, we have to solve a problem with $\vec{u}^{n+1} \cdot \nabla \vec{u}^{n+1}$ in (3.1) replaced by the lagged convection field $\vec{u}^{n} \cdot \nabla \vec{u}^{n+1}$. The corresponding weak problem is: compute $\vec{u}^{n+1}(\vec{x}, \omega) \in L^{2}\left(\Xi, \boldsymbol{H}_{E}^{1}(\Omega)\right)$ and $p^{n+1}(\vec{x}, \omega) \in$ $L^{2}\left(\Xi, L^{2}(\Omega)\right)$ satisfying

$$
\begin{array}{r}
\mathbb{E}\left[\nu(\xi(\omega))\left(\nabla \vec{u}^{n+1}, \nabla \vec{v}\right)\right]+\mathbb{E}\left[\left(\vec{u}^{n} \cdot \nabla \vec{u}^{n+1}, \vec{v}\right)\right]-\mathbb{E}\left[\left(p^{n+1}, \nabla \cdot \vec{v}\right)\right]=0, \\
\mathbb{E}\left[\left(q, \nabla \cdot \vec{u}^{n+1}\right)\right]=0,
\end{array}
$$

for all $\vec{v}(\vec{x}, \omega) \in L^{2}\left(\Xi, \boldsymbol{H}_{0}^{1}(\Omega)\right)$ and $q(\vec{x}, \omega) \in L^{2}\left(\Xi, L^{2}(\Omega)\right)$. This closely ressembles the deterministic weak problem $(2.3)-(2.4)$ but now the solutions and test functions are random fields and we have taken expectations. 
For the random variable $\xi$ in (3.9) the probability density function is $\rho(\lambda)=1 /(2 \sqrt{3})$ and $\Lambda:=\xi(\Xi)$, the range of $\xi$, is a finite interval. A key point for implementation is that for any measurable function $f$ of $\xi$, the expectation is given by the standard integral

$$
\mathbb{E}[f(\xi)]=\int_{\Lambda} \rho(\lambda) f(\lambda) d \lambda .
$$

Since $\nu=\nu(\xi(\omega)), \vec{u}$ and $p$ also depend on $\omega \in \Xi$ via $\xi$ and by defining $\lambda=\xi(\omega)$, we can transform (4.1)-(4.2) into the equivalent parametric formulation: find $\vec{u}^{n+1}(\vec{x}, \lambda) \in$ $\boldsymbol{V}_{E}(\Omega, \Lambda)$ and $p^{n+1}(\vec{x}, \lambda) \in W(\Omega, \Lambda)$ satisfying

$$
\begin{aligned}
& \int_{\Lambda} \rho(\lambda)\left\{\nu(\lambda)\left(\nabla \vec{u}^{n+1}, \nabla \vec{v}\right)+\left(\vec{u}^{n} \cdot \nabla \vec{u}^{n+1}, \vec{v}\right)-\left(p^{n+1}, \nabla \cdot \vec{v}\right)\right\} d \lambda=0, \\
& \int_{\Lambda} \rho(\lambda)\left(q, \nabla \cdot \vec{u}^{n+1}\right) d \lambda=0,
\end{aligned}
$$

for all $\vec{v}(\vec{x}, \lambda) \in \boldsymbol{V}_{0}(\Omega, \Lambda)$ and $q(\vec{x}, \lambda) \in W(\Omega, \Lambda)$. Note that $(\cdot, \cdot)$ in (4.3)-(4.4) represents the standard $L^{2}(\Omega)$ inner product. The spaces $V_{0}(\Omega, \Lambda)$ and $W(\Omega, \Lambda)$ now contain functions of the image coordinate $\lambda \in \Lambda$ and are defined as follows

$$
\begin{aligned}
\boldsymbol{V}_{0}(\Omega, \Lambda):=L_{\rho}^{2}\left(\Lambda, \boldsymbol{H}_{0}^{1}(\Omega)\right) & =\left\{v: \Omega \times \Lambda \rightarrow \mathbb{R} \mid \int_{\Lambda} \rho(\lambda)\|v\|_{\boldsymbol{H}_{0}^{1}(\Omega)}^{2} d \lambda<\infty\right\} \\
W(\Omega, \Lambda) & :=L_{\rho}^{2}\left(\Lambda, L^{2}(\Omega)\right)=\left\{w: \Omega \times \Lambda \rightarrow \mathbb{R} \mid \int_{\Lambda} \rho(\lambda)\|w\|_{L^{2}(\Omega)}^{2} d \lambda<\infty\right\}
\end{aligned}
$$

The solution space $\boldsymbol{V}_{E}(\Omega, \Lambda):=L_{\rho}^{2}\left(\Lambda, \boldsymbol{H}_{E}^{1}(\Omega)\right)$ is analogously defined and incorporates the essential (Dirichlet) boundary conditions on $\Gamma_{D}$.

Next, we derive a finite-dimensional Galerkin formulation by combining the stable spatial $Q_{2}-Q_{1}$ velocity-pressure approximation with standard polynomial approximation on $\Lambda$. To that end, let $S^{k}=S^{k}(\Lambda) \subset L_{\rho}^{2}(\Lambda)$ be the set of univariate polynomials in $\lambda$ of degree $\leq k$ on $\Lambda$. Then, the Picard linearization of the convection term requires us to compute the stochastic Galerkin solution $\vec{u}_{h k}^{n+1} \in \boldsymbol{X}_{E}^{h} \otimes S^{k} \subset \boldsymbol{V}_{E}(\Omega, \Lambda)$ and $p_{h k}^{n+1} \in M^{h} \otimes S^{k} \subset W(\Omega, \Lambda)$ satisfying the fully discrete formulation,

$$
\begin{aligned}
\mathbb{E}\left[\nu(\lambda)\left(\nabla \vec{u}_{h k}^{n+1}, \nabla \vec{v}_{h k}\right)\right]+\mathbb{E}\left[\left(\vec{u}_{h k}^{n} \cdot \nabla \vec{u}_{h k}^{n+1}, \vec{v}_{h k}\right)\right]-\mathbb{E}\left[\left(p_{h k}^{n+1}, \nabla \cdot \vec{v}_{h k}\right)\right] & =0, \\
\mathbb{E}\left[\left(\nabla \cdot \vec{u}_{h k}^{n+1}, q_{h k}\right)\right] & =0,
\end{aligned}
$$

for all $\vec{v}_{h k} \in \boldsymbol{X}_{0}^{h} \otimes S^{k}$ and $q_{h k} \in M^{h} \otimes S^{k}$. Alternatively, given $\vec{u}_{h k}^{n} \in \boldsymbol{X}_{E}^{h} \otimes S^{k}$ and $p_{h k}^{n} \in M^{h} \otimes S^{k}$, we can compute the updates $\vec{d}_{h k}^{n}:=\vec{u}_{h k}^{n+1}-\vec{u}_{h k}^{n} \in \boldsymbol{X}_{0}^{h} \otimes S^{k}$ and $\delta_{h k}^{n}:=p_{h k}^{n+1}-p_{h k}^{n} \in M^{h} \otimes S^{k}$ by solving the system

$$
\begin{aligned}
\mathbb{E}\left[\nu(\lambda)\left(\nabla \vec{d}_{h k}^{n}, \nabla \vec{v}_{h k}\right)\right]+\mathbb{E}\left[\left(\vec{u}_{h k}^{n} \cdot \nabla \vec{d}_{h k}^{n}, \vec{v}_{h k}\right)\right]-\mathbb{E}\left[\left(\delta_{h k}^{n}, \nabla \cdot \vec{v}_{h k}\right)\right] & =R_{n}\left(\vec{v}_{h k}\right), \\
\mathbb{E}\left[\left(\nabla \cdot \vec{d}_{h k}^{n}, q_{h k}\right)\right] & =r_{n}\left(q_{h k}\right),
\end{aligned}
$$

for all $\vec{v}_{h k} \in \boldsymbol{X}_{0}^{h} \otimes S^{k}$ and $q_{h k} \in M^{h} \otimes S^{k}$, where the residuals are given by

$$
\begin{aligned}
& R_{n}\left(\vec{v}_{h k}\right):=-\mathbb{E}\left[\nu(\lambda)\left(\nabla \vec{u}_{h k}^{n}, \nabla \vec{v}_{h k}\right)\right]-\mathbb{E}\left[\left(\vec{u}_{h k}^{n} \cdot \nabla \vec{u}_{h k}^{n}, \vec{v}_{h k}\right)\right]+\mathbb{E}\left[\left(p_{h k}^{n}, \nabla \cdot \vec{v}_{h k}\right)\right], \\
& r_{n}\left(q_{h k}\right):=-\mathbb{E}\left[\left(\nabla \cdot \vec{u}_{h k}^{n}, q_{h k}\right)\right] .
\end{aligned}
$$

If we now select a specific basis set $S^{k}=\left\{\varphi_{\ell}\right\}_{\ell=0}^{k}$ and expand the updates in terms of the tensor product basis functions,

$$
\vec{d}_{h k}^{n}(\vec{x}, \lambda)=\left[\begin{array}{c}
\sum_{\ell=0}^{k} \sum_{i=1}^{n_{u}} \alpha_{i \ell}^{x, n} \phi_{i}(\vec{x}) \varphi_{\ell}(\lambda) \\
\sum_{\ell=0}^{k} \sum_{i=1}^{n_{u}} \alpha_{i \ell}^{y, n} \phi_{i}(\vec{x}) \varphi_{\ell}(\lambda)
\end{array}\right], \quad \delta_{h k}^{n}(\vec{x}, \lambda)=\sum_{\ell=0}^{k} \sum_{j=1}^{n_{p}} \alpha_{j \ell}^{p, n} \psi_{j}(\vec{x}) \varphi_{\ell}(\lambda),
$$


the coefficient vectors $\boldsymbol{\alpha}^{u, n}=\left[\boldsymbol{\alpha}^{x, n}, \boldsymbol{\alpha}^{y, n}\right]$ and $\boldsymbol{\alpha}^{p, n}$ are computed by solving the saddlepoint system associated with (4.9)-(4.10). Specifically, we have,

$$
\left(\begin{array}{cc}
\mathbb{F}_{\nu}^{n} & \mathbb{B}^{T} \\
\mathbb{B} & 0
\end{array}\right)\left(\begin{array}{l}
\boldsymbol{\alpha}^{u, n} \\
\boldsymbol{\alpha}^{p, n}
\end{array}\right)=\left(\begin{array}{l}
\boldsymbol{f}^{u, n} \\
\boldsymbol{f}^{p, n}
\end{array}\right),
$$

where $\boldsymbol{f}^{u, n}$ and $\boldsymbol{f}^{p, n}$ are associated with the residuals $R_{n}$ and $r_{n}$ in (4.11)-(4.12). Note that this system has exactly the same saddle-point structure as (2.6).

4.1. Matrix components. The matrices $\mathbb{F}_{\nu}^{n}$ and $\mathbb{B}$ in (4.13) are Kronecker products of smaller matrices. To see this, we order the degrees of freedom to run over the basis functions for $S^{k}$ in turn. The update vectors $\boldsymbol{\alpha}^{x, n}$ and $\boldsymbol{\alpha}^{y, n}$ then each have $k+1$ consecutive blocks of length $n_{u}$, and $\boldsymbol{\alpha}^{p, n}$ has $k+1$ blocks of length $n_{p}$. We obtain

$$
\mathbb{B}:=\left[G_{0} \otimes B_{x}, G_{0} \otimes B_{y}\right],
$$

where $B_{x}, B_{y}$ are the component matrices defined in (2.7), and

$$
G_{0}:=\left[G_{0}\right]_{\ell s}=\mathbb{E}\left[\varphi_{s} \varphi_{\ell}\right], \quad \ell, s=0, \ldots, k .
$$

The structure of the convection-diffusion matrix $\mathbb{F}_{\nu}^{n}$ is more complicated:

$$
\mathbb{F}_{\nu}^{n}:=\underbrace{\left(\nu_{0} G_{0}+\nu_{1} G_{1}\right) \otimes \boldsymbol{A}}_{\text {diffusion part }}+\underbrace{\sum_{\ell=0}^{k} H_{\ell} \otimes \boldsymbol{N}_{\ell}}_{\text {convection part }} .
$$

However, there is a lot of structure in (4.16) to exploit. Indeed, $\boldsymbol{A}$ is the block-diagonal matrix representing the vector Laplacian operator with components defined in (2.9) and the Galerkin "G-matrix" $G_{1}$ takes the form,

$$
G_{1}:=\left[G_{1}\right]_{\ell s}=\mathbb{E}\left[\lambda \varphi_{s} \varphi_{\ell}\right], \quad \ell, s=0, \ldots, k .
$$

If we select a basis set $\left\{\varphi_{\ell}\right\}_{\ell=0}^{k}$ that is orthonormal with respect to the $L_{\rho}^{2}(\Lambda)$ inner product (scaled Legendre polynomials in the case of the uniform distribution), then $G_{0}$ is the identity matrix and $G_{1}$ is tridiagonal, with zeros on the main diagonal (see e.g., [11] and [6]). Fortunately, this makes the diffusion part of the matrix $\mathbb{F}_{\nu}^{n}$ in (4.13) block tridiagonal, and hence block sparse with sparse blocks.

On the other hand, the convection part of $\mathbb{F}_{\nu}^{n}$ is block dense with sparse blocks. It involves a sum of $(k+1)$ Kronecker products of the (dense) Galerkin "H-matrices",

$$
H_{\ell}:=\left[H_{\ell}\right]_{m s}=\mathbb{E}\left[\varphi_{\ell} \varphi_{s} \varphi_{m}\right], \quad m, s=0, \ldots, k,
$$

with the (sparse) convection matrices $\boldsymbol{N}_{\ell}$ that have block diagonal components,

$$
N_{\ell}\left(\vec{u}_{h k}^{n}\right):=\left[N_{\ell}\right]_{i j}=\left(\vec{u}_{h \ell}^{n} \cdot \nabla \phi_{i}, \phi_{j}\right), \quad i, j=1, \ldots, n_{u},
$$

(cf. (2.10)). The coefficients $\vec{u}_{h \ell}^{n}$ in (4.19) correspond to the $k+1$ spatial coefficients in the stochastic expansion of the lagged velocity field. To see this, note that at the $n$th iteration, the stochastic Galerkin velocity components can be expanded as follows

$$
u_{h k}^{x, n}(\vec{x}, \lambda):=\sum_{\ell=0}^{k} \underbrace{\sum_{i=1}^{n_{u}} u_{i \ell}^{x, n} \phi_{i}(\vec{x})}_{u_{h \ell}^{x, n}(\vec{x})} \varphi_{\ell}(\lambda), \quad u_{h k}^{y, n}(\vec{x}, \lambda):=\sum_{\ell=0}^{k} \underbrace{\sum_{i=1}^{n_{u}} u_{i \ell}^{y, n} \phi_{i}(\vec{x})}_{u_{h \ell}^{y, n}(\vec{x})} \varphi_{\ell}(\lambda),
$$

and we have

$$
\vec{u}_{h k}^{n}(\vec{x}, \lambda):=\sum_{\ell=0}^{k} \vec{u}_{h \ell}^{n}(\vec{x}) \varphi_{\ell}(\lambda), \quad \text { where } \quad \vec{u}_{h \ell}^{n}(\vec{x}):=\left[\begin{array}{c}
u_{h \ell}^{x, n}(\vec{x}) \\
u_{h \ell}^{y, n}(\vec{x})
\end{array}\right] .
$$


Similarly, the pressure solution has the expansion

$$
p_{h k}^{n}(\vec{x}, \lambda):=\sum_{\ell=0}^{k} p_{h \ell}^{n}(\vec{x}) \varphi_{\ell}(\lambda) .
$$

Our choice of orthonormal basis $\left\{\varphi_{\ell}\right\}_{\ell=1}^{k}$ for $S^{k}$ is often called a (generalized) Polynomial Chaos (PC) basis and the coefficients $\vec{u}_{h \ell}^{n}$ and $p_{h \ell}^{n}$ are known as PC coefficients. The usual scaling convention gives $\psi_{0}=1$ and since $\mathbb{E}\left[\psi_{\ell}\right]=0$ for $\ell>1$, the first-order statistics of the numerical solution are given by the leading PC coefficients,

$$
\mathbb{E}\left[\vec{u}_{h k}^{n}\right]=\vec{u}_{h 0}^{n}(\vec{x}), \quad \mathbb{E}\left[p_{h k}^{n}\right]=p_{h 0}^{n}(\vec{x}) .
$$

Before discussing how to solve the saddle-point systems (4.13), we present some numerical results for the backward-facing step problem.

4.2. Flow over a backward-facing step test problem. Mirroring the setup in Section 2 , we choose $\mathbb{E}[\nu]=\nu_{0}=1 / 50, d=1$ and $L=5$. Below, we show results for the specific case $\nu_{1}=1 / 500$, which yields $\nu \sim U[0.01654,0.02346]$ and $\mathbb{E}[\operatorname{Re}] \approx 67$. For the spatial discretization, we use the same grid as in Figure 2.3 and for the stochastic approximation, we use polynomials of degree $k=5$. The numerical solution is generated by solving the Picard systems (4.7)-(4.8) (starting from an initial guess provided by the stochastic Stokes-flow solution; see Section 5.3). The statistical properties of the converged solution (obtained after 15 Picard steps) are illustrated in Figures 4.1-4.5.
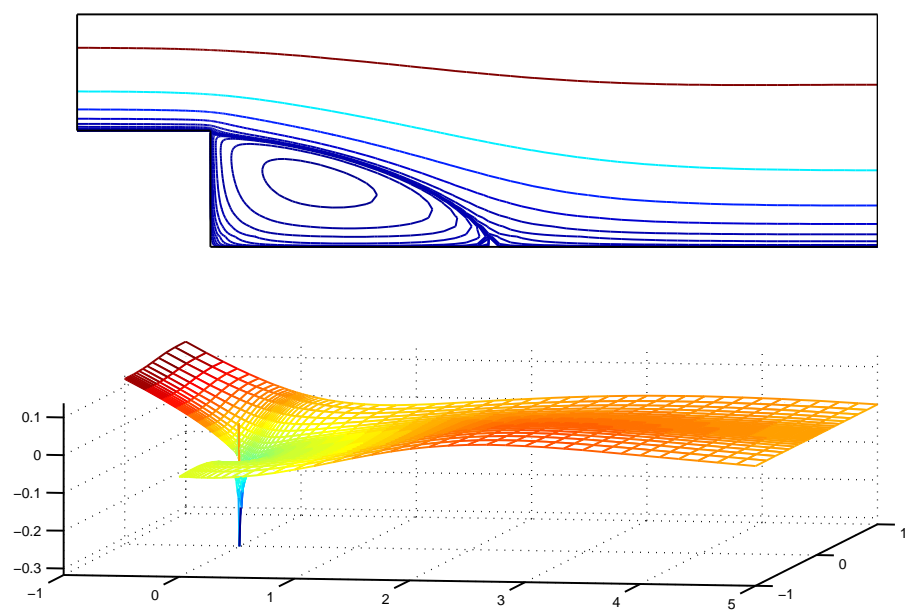

FIG. 4.1. Streamlines of the mean velocity $\mathbb{E}\left[\vec{u}_{h k}^{15}\right]$ (top) and mean pressure $\mathbb{E}\left[p_{h k}^{15}\right]$ (bottom).

As anticipated, the mean solution in Figure 4.1 looks exactly like the deterministic solution shown in Figure 2.3. The spatial singularity in the pressure solution is still a key feature. The variance of the pressure, $\operatorname{Var}\left[p_{h k}^{15}\right]$, and the variance of the magnitude of the velocity, $\operatorname{Var}\left[\left|\vec{u}_{h k}^{15}\right|\right]$, are plotted in Figure 4.2. Modelling viscosity as a random variable is equivalent to having uncertainty in the volume of fluid flow at the inflow. This generates uncertainty in the length of the recirculating eddy, so the variance of the velocity is concentrated in two areas downstream of the step. In contrast, the pressure uncertainty is concentrated at the inflow - the uncertainty in the volume of fluid at the inflow generates uncertainty in the pressure drop between the inflow and outflow (recall that the outflow boundary condition forces the pressure to have mean zero). 

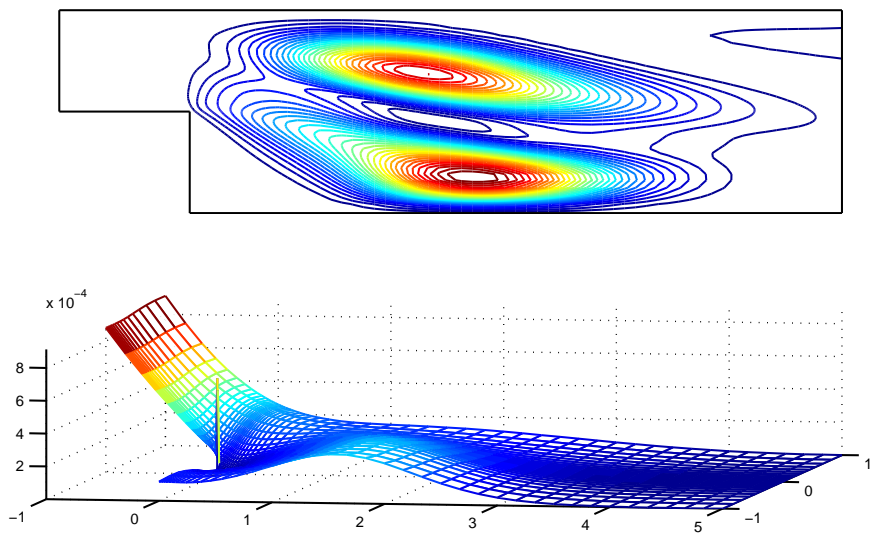

FIG. 4.2. Variance of the pressure, $\operatorname{Var}\left[p_{h k}^{15}\right]$ (bottom) and variance of the magnitude of the velocity, $\operatorname{Var}\left[\left|\vec{u}_{h k}^{15}\right|\right]$ (top). (The maximum value of the peaks in the top plot is 6.4406e-04).
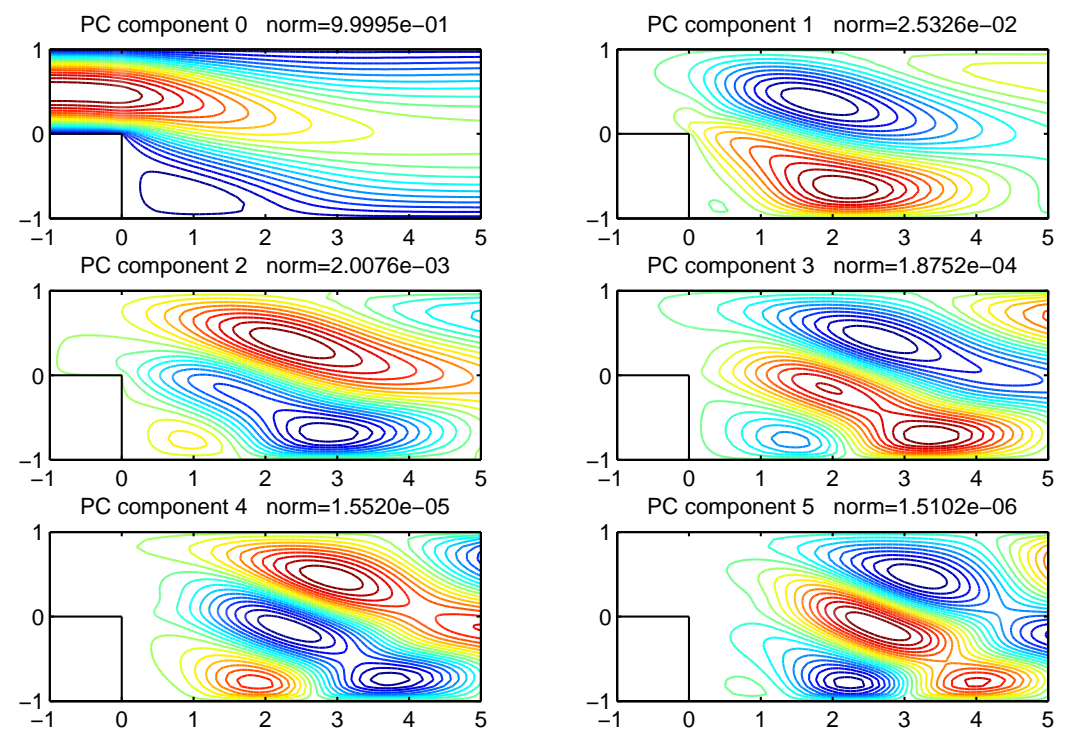

FIG. 4.3. Contours of the PC coefficients $u_{h \ell}^{x, 15}(\vec{x}), \ell=0,1, \ldots 5$, of the horizontal velocity component, $u_{h k}^{x, 15}$. The norms $\left\|u_{h \ell}^{x, 15}(\vec{x})\right\|_{L^{\infty}(\Omega)}$ are also displayed, and decay as the index $\ell$ increases.

The influence of the polynomial degree, $k$, on the numerical solution can be assessed by looking at the PC coefficients in (4.20)-(4.21). The scalar coefficients of the horizontal velocity component, $u_{h k}^{x, 15}$, of the converged velocity solution are plotted in Figure 4.3. The coefficients are spatially smooth and the flow resolution increases with increasing polynomial degree (i.e., the linear component has three "peaks", whereas the quartic component has six "peaks"). An important point for our solver (see Lemmas 5.2-5.3) is that the quantity $\left\|u_{h \ell}^{x, 15}(\vec{x})\right\|_{L^{\infty}(\Omega)}$ decreases rapidly with increasing $\ell$. Note also that the PC coefficients corresponding to $\ell \geq 1$ are not zero, so the velocity solution depends on the stochastic variable (unlike the Stokes velocity). The PC coefficients of the converged pressure solution are also plotted in Figure 4.4. Notice that the coefficients of $\varphi_{0}$ and $\varphi_{1}$ are dominant, suggesting the pressure depends almost linearly on $\lambda$. 
PC component 0
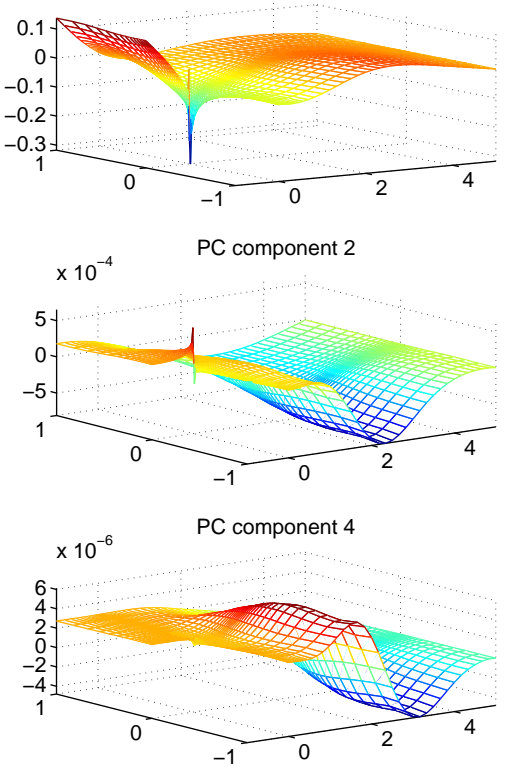

PC component 1

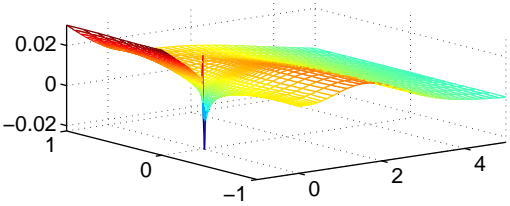

PC component 3
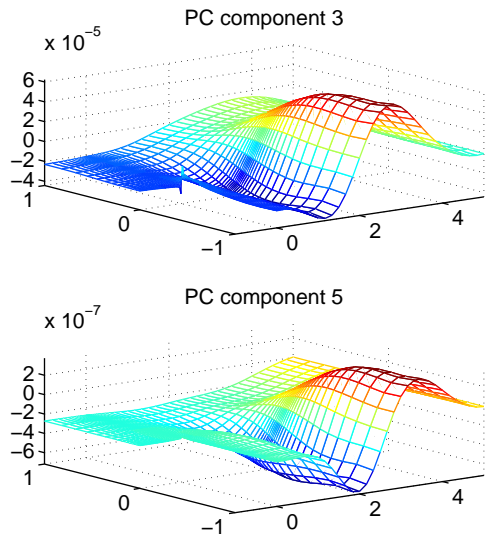

FIG. 4.4. PC coefficients $p_{h \ell}^{x, 15}(\vec{x}), \ell=0,1, \ldots 5$, of the pressure solution, $p_{h k}^{x, 15}$.

The above observations suggest that, for our test problem, solution statistics can be accurately estimated using low polynomial degrees, $k$. Consider the vorticity,

$$
\omega(\vec{x}, \lambda):=\frac{\partial}{\partial x}\left(u^{y}(\vec{x}, \lambda)\right)-\frac{\partial}{\partial y}\left(u^{x}(\vec{x}, \lambda)\right) .
$$

Replacing the velocity by $\vec{u}_{h k}^{15}$ in (4.23) and expanding, yields the approximate vorticity,

$$
\omega_{h k}^{15}(\vec{x}, \lambda):=\sum_{\ell=0}^{k} \underbrace{\frac{\partial}{\partial x}\left(u_{h \ell}^{y, 15}(\vec{x}, \lambda)\right)-\frac{\partial}{\partial y}\left(u_{h \ell}^{x, 15}(\vec{x}, \lambda)\right)}_{=\omega_{\ell}(\vec{x})} \varphi_{\ell}(\lambda) .
$$

The vorticity along the lower channel wall $[0,5]$ is an important quantity of interest. When $\nu_{0}=1 / 50$, the length of the recirculating eddy in the mean flow is close to two and it is interesting to study the total vorticity along the wall segment $[1,3]$, i.e.,

$$
Q(\lambda):=\int_{1}^{3} \omega_{h k}^{15}(\vec{x}, \lambda) d s .
$$

Approximations to the standard deviation, $\operatorname{stdv}(Q)$, computed with different polynomial degrees $k$, are recorded in Table 4.1. Observe that $\operatorname{stdv}(Q)$ grows linearly with the standard deviation of the viscosity, $\nu_{1}$. The degree of polynomial needed to approximate $\operatorname{stdv}(Q)$ to four decimal places increases as we increase $\nu_{1}$. In general, we observe that $k \leq 5$ suffices for expected Reynolds numbers of up to two hundred (see Table 3.1). When $\nu_{1}=1 / 500$, there is very little uncertainty in $\nu$ and we actually only need $k=2$.

For higher Reynolds numbers, slightly higher polynomials degrees are needed to accurately capture $\operatorname{stdv}(Q)$. Fixing $\nu_{0}=1 / 200$ and varying $\nu_{1}$ to produce expected Reynolds numbers in the range $[200,330]$, leads to mean flow fields with a much longer recirculating eddy. The estimated standard deviations of the total vorticity on the wall 


\begin{tabular}{l|rrrrr}
\hline & $k=1$ & $k=2$ & $k=3$ & $k=4$ & $k=5$ \\
\hline$\nu_{1}=\nu_{0} / 10$ & 0.2390 & 0.2392 & $*$ & $*$ & $*$ \\
$\nu_{1}=2 \nu_{0} / 10$ & 0.4769 & 0.4768 & 0.4762 & $*$ & $*$ \\
$\nu_{1}=3 \nu_{0} / 10$ & 0.7093 & 0.6992 & 0.6942 & 0.6941 & $*$ \\
$\nu_{1}=4 \nu_{0} / 10$ & 0.9257 & 0.8683 & 0.8510 & 0.8525 & 0.8526 \\
\hline
\end{tabular}

TABLE 4.1

Standard deviation of $Q$ in (4.25) for fixed mean viscosity $\nu_{0}=1 / 50$ and varying $\nu_{1}$. The symbol * indicates the approximation has converged to 4 decimal places.

segment $[3,7]$ (computed by increasing the channel length to $L=15$ ) are recorded in Table 4.2. The spatial contributions $\omega_{\ell}$ in (4.24) to the estimated vorticity on the entire lower channel wall $[0,15]$, computed with $k=5$, are also plotted in Figure 4.5.

\begin{tabular}{l|rrrrr}
\hline & $k=1$ & $k=2$ & $k=3$ & $k=4$ & $k=5$ \\
\hline$\nu_{1}=\nu_{0} / 10$ & 0.6553 & 0.6492 & 0.6491 & $*$ & $*$ \\
$\nu_{1}=2 \nu_{0} / 10$ & 1.2639 & 1.2150 & 1.2139 & 1.2138 & $*$ \\
$\nu_{1}=3 \nu_{0} / 10$ & 1.7752 & 1.6197 & 1.6180 & 1.6167 & 1.6162 \\
$\nu_{1}=4 \nu_{0} / 10$ & 2.1379 & 1.8133 & 1.8320 & 1.8287 & 1.8291 \\
\hline \multicolumn{5}{c}{ TABLE 4.2}
\end{tabular}

Standard deviation of $Q$ in $(4.25)$ (with $[1,3]$ replaced by $[3,7]$ ), for fixed mean viscosity $\nu_{0}=1 / 200$ and varying $\nu_{1}$. The symbol $*$ indicates the approximation has converged to 4 decimal places.
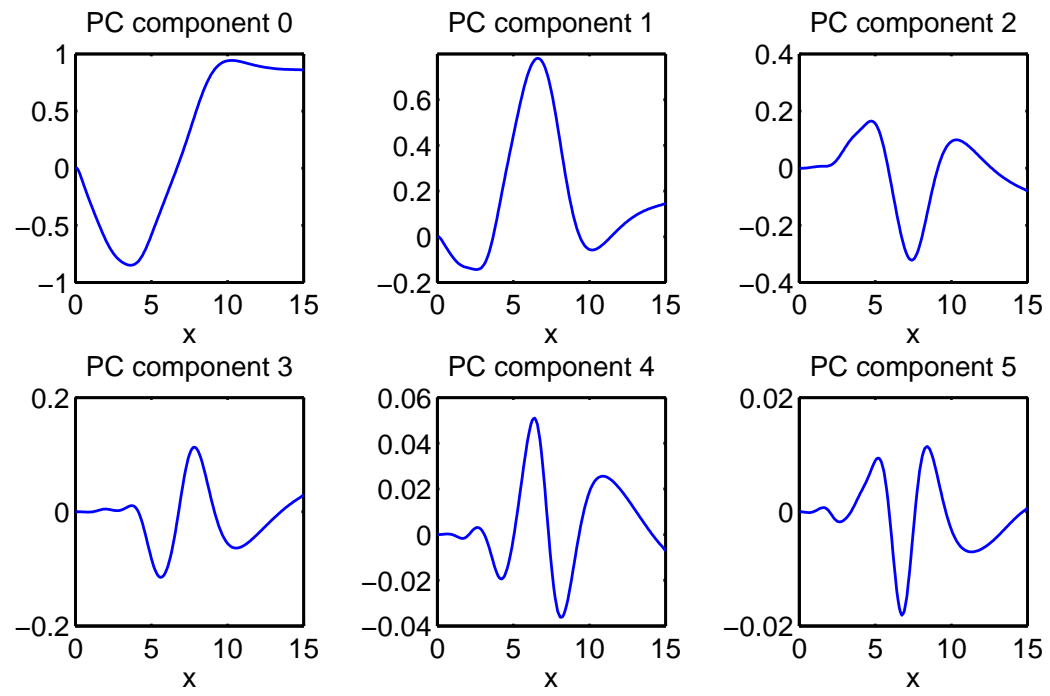

FIG. 4.5. Spatial components $\omega_{\ell}, \ell=0,1, \ldots, 5$, of the vorticity distribution along the bottom wall of the channel, $\{-1\} \times[0,15]$, for $\nu_{0}=1 / 200, \nu_{1}=3 / 2000$ and $k=5$.

The fact that we don't need to choose $k$ to be very large (at least for problems with $\mathbb{E}[\operatorname{Re}] \leq 330)$ is advantageous for the linear algebra, since the dimension of the system (4.13) is a factor of $k+1$ larger than (2.6), and the number of blocks in the Kronecker product matrices is then guaranteed to be small. The issue of how to balance the spatial error (depending on $h$ ) and the spectral error (depending on $k$ ) in stochastic Galerkin approximation is currently an active research topic, (e.g., see Xiu [15, Chapter 6] and Le Maître \& Knio [10, Chapter 9] for further discussion). 
5. Preconditioned GMRES. We now focus on the solution of the linear systems (4.13) and develop new preconditioners for use with GMRES. Our aim is to generalize the preconditioner (2.11) to the stochastic problem. Although the new saddle-point systems have increased dimensionality (i.e., there are now $\left(2 n_{u}+n_{p}\right)(k+1)$ equations) and a more complex Kronecker product structure, right-preconditioned GMRES still converges in two iterations if the ideal preconditioner (2.11) is applied to (4.13). In the sequel, we will drop the superscript $n$ and consider a single system. To build a practical preconditioner, we need two ingredients: an approximation $\mathbb{P}_{F}$ to the convection-diffusion matrix $\mathbb{F}_{\nu}$ in (4.16); and an approximation $\mathbb{P}_{S}$ to the Schur complement $\mathbb{S}:=\mathbb{B F}_{\nu}^{-1} \mathbb{B}^{T}$. The resulting exact preconditioner is then

$$
\mathscr{P}_{E}:=\left(\begin{array}{cc}
\mathbb{P}_{F} & \mathbb{B}^{T} \\
0 & -\mathbb{P}_{S}
\end{array}\right)
$$

As usual, we require the approximations to be robust with respect to the problem parameters: i.e., the discretization parameters $h$ and $k$, and the statistical parameters $\nu_{0}$ and $\nu_{1}$, which control the distribution of $\operatorname{Re}(\omega)$. Furthermore, solves with $\mathbb{P}_{F}$ and $\mathbb{P}_{S}$ must have low complexity. Herein, we add the extra criterion that deterministic solvers be exploited wherever possible. Balancing these requirements is a tough challenge.

5.1. Approximating the convection-diffusion matrix. The coefficient matrix $\mathbb{F}_{\nu}$ in (4.16) is non-symmetric. However, the diffusion part is always symmetric and if $\mathbb{P}(\nu(\omega)>0)=1$, then it is also positive definite.

LEMMA 5.1. If $\nu$ is of the form (3.9) with $\xi \sim U(-\sqrt{3}, \sqrt{3})$ and (3.12) holds, the diffusion matrix $\mathbb{D}:=\left(\nu_{0} I+\nu_{1} G_{1}\right) \otimes \boldsymbol{A}$ is symmetric and positive definite. Moreover, if $\mathscr{T}_{h}$ is shape regular and quasi uniform, the eigenvalues lie in the bounded interval

$$
\left[c \nu_{\min } h^{2}, C \nu_{\max }\right]
$$

where $c$ and $C$ are constants independent of $h, k$ and $\nu$, and $\nu_{\min }, \nu_{\max }$ are as in (3.10).

Proof. Since $G_{1}$ and $\boldsymbol{A}$ are symmetric by (4.17) and (2.9), we have

$$
\mathbb{D}^{T}=\left(\nu_{0} I+\nu_{1} G_{1}^{T}\right) \otimes \boldsymbol{A}^{T}=\mathbb{D} .
$$

The eigenvalues of $\mathbb{D}$ are products of eigenvalues of $\left(\nu_{0} I+\nu_{1} G_{1}\right)$ and eigenvalues of $\boldsymbol{A}$. Denote the (positive) extremal eigenvalues of the symmetric positive definite matrix $\boldsymbol{A}$ by $\sigma_{\min }(\boldsymbol{A})$ and $\sigma_{\max }(\boldsymbol{A})$. Let $\boldsymbol{w} \in \mathbb{R}^{k+1} \backslash\{\boldsymbol{O}\}$ and define $w(\lambda):=\sum_{\ell=0}^{k} w_{i} \varphi_{\ell}(\lambda) \in S^{k}$. We have $\boldsymbol{w}^{T} \boldsymbol{w}=\mathbb{E}\left[w^{2}\right]$ and $\boldsymbol{w}^{T}\left(\nu_{0} I+\nu_{1} G_{1}\right) \boldsymbol{w}=\mathbb{E}\left[\nu w^{2}\right]$. Using (3.10), the eigenvalues of $\left(\nu_{0} I+\nu_{1} G_{1}\right)$ belong to the interval $\left[\nu_{\min }, \nu_{\max }\right]$. The eigenvalues of $\mathbb{D}$ must then lie in the interval $\left[\nu_{\min } \sigma_{\min }(\boldsymbol{A}), \nu_{\max } \sigma_{\max }(\boldsymbol{A})\right]$. Positive definiteness is assured by (3.12) and (5.2) follows from the standard bounds for the eigenvalues of the $Q_{2}$ (or $Q_{1}$ ) finite element matrix $\boldsymbol{A}$ (e.g. see [4, Theorem 5.21]).

In Section 6, we present results for three versions of the preconditioner (5.1), all of which are based on the same approximation $\mathbb{P}_{F}$ to the convection-diffusion matrix $\mathbb{F}_{\nu}$. At first glance, $\mathbb{P}_{F}=\mathbb{D}$ seems a good candidate. Indeed, since

$$
\mathbb{D}^{-1}=\left(\nu_{0} I+\nu_{1} G_{1}\right)^{-1} \otimes \boldsymbol{A}^{-1},
$$

solves with $\mathbb{D}$ can be performed via $(k+1)$ solves with $\boldsymbol{A}$ (for which fast solvers, e.g. AMG, [1], are available) and $2 n_{u}$ solves with $\left(\nu_{0} I+\nu_{1} G_{1}\right)$ (which is small and tridiagonal). By adapting [14, Theorem 4.1], we can prove the following result.

LEMMA 5.2. The generalized field of values of the matrix $\mathbb{F}_{\nu}$ associated with the lagged velocity $\vec{u}_{h k}$, with respect to the preconditioner $\mathbb{D}$, is contained in the circle

$$
\left\{z \in \mathbb{C}:|z-1| \leq \frac{2 C_{\Omega} \tau_{k}}{\nu_{\min }}\right\}, \quad \tau_{k}:=\left\|\mathbb{E}\left[\vec{u}_{h k}\right]\right\|_{L^{\infty}(\Omega)}+\gamma_{k} \sum_{\ell=1}^{k}\left\|\vec{u}_{h \ell}\right\|_{L^{\infty}(\Omega)},
$$

where the constant $C_{\Omega}$ depends only on the spatial domain, $\nu_{\text {min }}$ is defined in (3.10), $\gamma_{k}$ depends only on $k$, and $\vec{u}_{h \ell}$ is the $\ell$ th PC coefficient of the lagged velocity solution. 
Proof. The field of values of interest is the set of complex numbers

$$
\operatorname{FOV}\left(\mathbb{F}_{\nu}, \mathbb{D}\right):=\left\{\frac{\boldsymbol{v}^{H} \mathbb{F}_{\nu} \boldsymbol{v}}{\boldsymbol{v}^{H} \mathbb{D} \boldsymbol{v}}, \quad \boldsymbol{v} \in \mathbb{C}^{2 n_{u}(k+1)} \backslash\{\boldsymbol{o}\}\right\} .
$$

Defining $G:=\nu_{0} I+\nu_{1} G_{1}$ and denoting the convection part of $\mathbb{F}_{\nu}$ by $\mathbb{N}$, we have,

$$
\frac{\boldsymbol{v}^{H} \mathbb{F}_{\nu} \boldsymbol{v}}{\boldsymbol{v}^{H} \mathbb{D} \boldsymbol{v}}=1+\frac{\boldsymbol{v}^{H} \mathbb{N} \boldsymbol{v}}{\boldsymbol{v}^{H} \mathbb{D} \boldsymbol{v}}=1+\sum_{\ell=0}^{k} \frac{\boldsymbol{v}^{H}\left(H_{\ell} \otimes N_{\ell}\right) \boldsymbol{v}}{\boldsymbol{v}^{H}(G \otimes \boldsymbol{A}) \boldsymbol{v}}=1+\sum_{\ell=0}^{k}\left(\frac{\boldsymbol{u}^{H} H_{\ell} \boldsymbol{u}}{\boldsymbol{u}^{H} G \boldsymbol{u}}\right)\left(\frac{\boldsymbol{w}^{H} N_{\ell} \boldsymbol{w}}{\boldsymbol{w}^{H} \boldsymbol{A} \boldsymbol{w}}\right),
$$

where $\boldsymbol{v}=\boldsymbol{u} \otimes \boldsymbol{w}$, with $\boldsymbol{u} \in \mathbb{C}^{k+1}$ and $\boldsymbol{w} \in \mathbb{C}^{2 n_{u}}$. For normal matrices $C$, the field of values is $\operatorname{conv}(\lambda(C))$, the convex hull of the set of eigenvalues. Following [14, Theorem 4.1] and noting that $G$ and $H_{\ell}$ are symmetric, leads to

$$
\operatorname{FOV}\left(\mathbb{F}_{\nu}, \mathbb{D}\right)-1 \subset \sum_{\ell=0}^{k} \operatorname{conv}\left(\operatorname{conv}\left(\lambda\left(H_{\ell}, G\right)\right) \cdot \operatorname{FOV}\left(\boldsymbol{N}_{\ell}, \boldsymbol{A}\right)\right)
$$

In (5.5), $\lambda\left(H_{\ell}, G\right)$ is the set of $(k+1)$ (real) values $\lambda_{\ell}$ satisfying $H_{\ell} \boldsymbol{x}=\lambda_{\ell} G \boldsymbol{x}$. Since the eigenvalues of $G$ belong to the interval $\left[\nu_{\min }, \nu_{\max }\right]$ and $H_{0}=I$,

$$
\lambda\left(H_{0}, G\right) \subset\left[1 / \nu_{\max }, 1 / \nu_{\min }\right] .
$$

Each entry of $H_{\ell}$ is a weighted integral of a polynomial of maximum degree $2 k+\ell$. For a fixed $\ell$, let $S_{N}:=\left\{\eta_{1}, \ldots, \eta_{N}\right\} \subset(-\sqrt{3}, \sqrt{3})$ denote the nodes of the $N$-point Gauss-Legendre quadrature rule. With $N:=k+\left\lceil\frac{\ell+1}{2}\right\rceil$, the rule is exact for all entries of $H_{\ell}$. In [6] it is shown that the eigenvalues of $H_{\ell}$ must then lie in $\left[\alpha_{\ell}, \beta_{\ell}\right]$ where,

$$
\alpha_{\ell}:=\min _{\eta_{i} \in S_{N}}\left\{\psi_{\ell}\left(\eta_{i}\right)\right\}, \quad \beta_{\ell}:=\max _{\eta_{i} \in S_{N}}\left\{\psi_{\ell}\left(\eta_{i}\right)\right\}, \quad \ell=1, \ldots, k .
$$

Herein, for $\xi \sim U(-\sqrt{3}, \sqrt{3})$, our normalized basis functions for $S^{k}$ are defined via $\psi_{\ell}(\lambda):=\sqrt{2 \ell+1} L_{\ell}(x / \sqrt{3})$, where $\left\{L_{\ell}(x)\right\}$ are the standard Legendre polynomials of degree $\ell$ on $[-1,1]$. Since $\left|L_{\ell}(x)\right| \leq 1$ for $x \in[-1,1]$, it follows that

$$
\left|\psi_{\ell}(\lambda)\right| \leq \sqrt{2 \ell+1}, \quad \forall \lambda \in[-\sqrt{3}, \sqrt{3}],
$$

and the eigenvalues of each $H_{\ell}$ lie in $\left[-\gamma_{k}, \gamma_{k}\right]$, where $\gamma_{k}:=\sqrt{2 k+1}$. Hence, $\lambda\left(H_{\ell}, G\right) \subset$ $\left[-\gamma_{k} / \nu_{\max }, \gamma_{k} / \nu_{\min }\right]$ and any $z \in \operatorname{conv}\left(\lambda\left(H_{\ell}, G\right)\right)$ satisfies $|z| \leq \gamma_{k} / \nu_{\min }$ for $\ell \geq 1$.

It remains to find bounds for the complex values $\operatorname{FOV}\left(\boldsymbol{N}_{\ell}, \boldsymbol{A}\right)$. Extending the argument in [14, Theorem 4.1] (which is for scalar convection-diffusion matrices and different convection coefficients), we can show that for every $z \in \operatorname{FOV}\left(\boldsymbol{N}_{\ell}, \boldsymbol{A}\right)$,

$$
|z| \leq 2 C_{\Omega}\left\|\vec{u}_{h \ell}\right\|_{L^{\infty}(\Omega)}, \quad \ell=0,1, \ldots, k,
$$

where $C_{\Omega}$ is the Poincaré constant for the spatial domain and $\vec{u}_{h l}$ is the $\ell$ th PC coefficient of the lagged velocity. Combining all these bounds with (5.5) gives the result.

REMARK 5.1. The rate of residual reduction of GMRES is bounded by a computable (asymptotic) convergence factor whenever the field of values is contained in an ellipse in the right-half plane - that is, whenever the circle in (5.3) excludes the origin. The bound (5.3) suggests that if $\mathbb{F}_{\nu}$ is preconditioned using the diffusion matrix $\mathbb{D}$ then the rate of convergence of GMRES will be inversely proportional to $\nu_{\min }$. Such a deterioration is clearly evident in actual computations (see Table 5.1, later).

REMARK 5.2. The bound $\gamma_{k}=\sqrt{2 k+1}$, for the eigenvalues of the $H_{\ell}$ matrices in the above proof, is somewhat pessimistic. For our specific test problem, the values $\left\|\vec{u}_{h \ell}\right\|_{L^{\infty}(\Omega)}$ also decay rapidly as $\ell$ (and hence $k$ ) increases (e.g. see Figure 4.3) and so the constant $\tau_{k}$ in Lemma 5.2 is almost independent of the value of $k$. 
The radius of the circle containing the generalized field of values in Lemma 5.2 is bounded independently of the parameter $h$. For $\nu_{0}=O(1)$, we have $\nu_{\min }=O(1)$ and in that case, the preconditioner $\mathbb{P}_{F}=\mathbb{D}$ is likely to be effective. On the other hand, if we want to solve flow problems with small viscosity values (i.e., high Reynolds numbers) then we need to look at the convection-diffusion matrix again. Noting that $G_{0}=H_{0}=I$, and $G_{1}=H_{1}$ (since $\psi_{0}=1$ and $\psi_{1}=\lambda$ ), we can rearrange $\mathbb{F}_{\nu}$ as follows

$$
\mathbb{F}_{\nu}=I \otimes \underbrace{\left(\nu_{0} \boldsymbol{A}+\boldsymbol{N}_{0}\right)}_{\boldsymbol{F}_{0}}+G_{1} \otimes \underbrace{\left(\nu_{1} \boldsymbol{A}+\boldsymbol{N}_{1}\right)}_{\boldsymbol{F}_{1}}+\sum_{\ell=2}^{k} H_{\ell} \otimes \boldsymbol{N}_{\ell} .
$$

The leading term is now

$$
\mathbb{F}_{0}:=I \otimes\left(\nu_{0} \boldsymbol{A}+\boldsymbol{N}_{0}\right):=I \otimes \boldsymbol{F}_{0},
$$

where $\boldsymbol{F}_{0}$ is the spatial convection-diffusion operator, with diffusion coefficient $\nu_{0}$, and convection coefficient given by $\vec{u}_{h 0}$, the mean lagged velocity field. The second term in (5.8) is also associated with a spatial convection-diffusion operator $\boldsymbol{F}_{1}$, with coefficients $\nu_{1}$ and $\vec{u}_{h 1}$. In the sequel, we call $\mathbb{F}_{0}$ the mean convection-diffusion matrix and since it is a block-diagonal version of the deterministic scalar convection-diffusion operator $F_{0}$, it is natural to consider $\mathbb{P}_{F}=\mathbb{F}_{0}$. This new approximation is non-symmetric. The next result gives the analogue of Lemma 5.2 and follows the proof of [14, Theorem 4.3].

LEMMA 5.3. The generalized (complex) eigenvalues of the matrix $\mathbb{F}_{\nu}$ associated with the lagged velocity $\vec{u}_{h k}$, with respect to the matrix $\mathbb{F}_{0}$, are contained in the circle

$$
\left\{z \in \mathbb{C}:|z-1| \leq 2 C_{\Omega} \delta_{k}\right\}, \quad \delta_{k}:=\frac{\nu_{1} \sqrt{3}}{\nu_{0}}+\frac{\gamma_{k}}{\nu_{0}} \sum_{\ell=1}^{k}\left\|\vec{u}_{h \ell}(\vec{x})\right\|_{L^{\infty}(\Omega)},
$$

where the constant $C_{\Omega}$ depends only on the spatial domain, $\nu_{0}$ and $\nu_{1}$ are the mean and standard deviation of the viscosity, respectively, $\delta_{k}$ depends only on the polynomial degree $k$, and $\vec{u}_{h \ell}$ is the $\ell$ th PC coefficient of the lagged velocity solution.

Proof. As in Lemma 5.2, let $\boldsymbol{v}=\boldsymbol{u} \otimes \boldsymbol{w}$, with $\boldsymbol{u} \in \mathbb{C}^{k+1}$ and $\boldsymbol{w} \in \mathbb{C}^{2 n_{u}}$. This time,

$$
\begin{aligned}
\frac{\boldsymbol{v}^{H} \mathbb{F}_{\nu} \boldsymbol{v}}{\boldsymbol{v}^{H} \mathbb{F}_{0} \boldsymbol{v}} & =1+\nu_{1} \frac{\boldsymbol{v}^{H}\left(G_{1} \otimes \boldsymbol{A}\right) \boldsymbol{v}}{\boldsymbol{v}^{H}\left(I \otimes \boldsymbol{F}_{0}\right) \boldsymbol{v}}+\sum_{\ell=1}^{k} \frac{\boldsymbol{v}^{H}\left(H_{\ell} \otimes N_{\ell}\right) \boldsymbol{v}}{\boldsymbol{v}^{H}\left(I \otimes \boldsymbol{F}_{0}\right) \boldsymbol{v}} \\
& =1+\nu_{1}\left(\frac{\boldsymbol{u}^{H} G_{1} \boldsymbol{u}}{\boldsymbol{u}^{H} \boldsymbol{u}}\right)\left(\frac{\boldsymbol{w}^{H} \boldsymbol{A} \boldsymbol{w}}{\boldsymbol{w}^{H} \boldsymbol{F}_{0} \boldsymbol{w}}\right)+\sum_{\ell=1}^{k}\left(\frac{\boldsymbol{u}^{H} H_{\ell} \boldsymbol{u}}{\boldsymbol{u}^{H} \boldsymbol{u}}\right)\left(\frac{\boldsymbol{w}^{H} N_{\ell} \boldsymbol{w}}{\boldsymbol{w}^{H} \boldsymbol{F}_{0} \boldsymbol{w}}\right)
\end{aligned}
$$

The (real) eigenvalues of $G_{1}$ belong to $[-\sqrt{3}, \sqrt{3}]$ and the (real) eigenvalues of each $H_{\ell}$ are contained in $\left[-\gamma_{k}, \gamma_{k}\right]$. From Lemma 5.2, we further deduce that

$$
\left|\boldsymbol{w}^{H} \boldsymbol{N}_{\ell} \boldsymbol{w}\right| \leq 2 C_{D}\left\|\vec{u}_{h \ell}\right\|_{L^{\infty}(\Omega)} \boldsymbol{w}^{H} \boldsymbol{A} \boldsymbol{w}=2 C_{D} \nu_{0}^{-1}\left\|\vec{u}_{h \ell}\right\|_{L^{\infty}(\Omega)} \boldsymbol{w}^{H}\left(\nu_{0} \boldsymbol{A}\right) \boldsymbol{w} .
$$

Noting that the (block-diagonal) matrix $\boldsymbol{N}_{0}$ is positive semi-definite, gives the bound,

$$
\left|\boldsymbol{w}^{H} \boldsymbol{F}_{0} \boldsymbol{w}\right|=\left|\boldsymbol{w}^{H}\left(\nu_{0} \boldsymbol{A}+\boldsymbol{N}_{0}\right) \boldsymbol{w}\right| \geq \nu_{0} \boldsymbol{w}^{H} \boldsymbol{A} \boldsymbol{w} .
$$

Combining these two results yields

$$
\left|\boldsymbol{w}^{H} \boldsymbol{N}_{\ell} \boldsymbol{w}\right| \leq 2 C_{D} \nu_{0}^{-1}\left\|\vec{u}_{h \ell}\right\|_{L^{\infty}(\Omega)}\left|\boldsymbol{w}^{H} \boldsymbol{F}_{0} \boldsymbol{w}\right|
$$

and the result immediately follows.

REMARK 5.3. The asymptotic rate of convergence of GMRES is bounded whenever the eigenvalues lie in a circle in the right-half plane. In Lemma 5.3, the bound for the radius of the circle depends on the ratio $\nu_{1} / \nu_{0}$, as opposed to the minimum possible value of the viscosity, $\nu_{\min }$. Hence, we anticipate that $\mathbb{F}_{0}$ provides a better preconditioner than $\mathbb{D}$ for the convection-diffusion matrix when $\nu_{0}<<1$. 
We now have a choice of two preconditioners for $\mathbb{F}_{\nu}$. Lemmas 5.2 and 5.3 suggest that the mean convection-diffusion approximation $\mathbb{F}_{0}$ is more robust with respect to the viscosity than the diffusion approximation $\mathbb{D}$. To corroborate this theory, we now present some results using right-preconditioned GMRES to solve a system of equations with coefficient matrix $\mathbb{F}_{\nu}$ (corresponding to the final, Picard step) for varying $h, k, \nu_{0}$ and $\nu_{1}$. The stopping tolerance for the relative residual error is set to $10^{-6}$ and iteration counts are recorded in Table 5.1. As predicted, both approximations are robust with respect to the discretization parameters $h$ and $k$ and the diffusion preconditioner $\mathbb{D}$ is the best when $\nu_{0}=1$. Iteration counts increase as $\nu_{\min } \rightarrow 0$, in both cases, but the degradation is far less for the mean convection-diffusion preconditioner, $\mathbb{F}_{0}$.

\begin{tabular}{l|l|l|ccc|ccc}
\hline $\mathbb{P}_{F}$ & $\nu_{0}$ & & $k=2$ & 4 & 6 & $k=2$ & 4 & 6 \\
\hline \multirow{3}{*}{$\mathbb{D}$} & \multirow{3}{*}{1} & $\nu_{1}=\nu_{0} / 10$ & 6 & 6 & 6 & 6 & 6 & 6 \\
& & $\nu_{1}=2 \nu_{0} / 10$ & 6 & 7 & 7 & 6 & 7 & 7 \\
& & $\nu_{1}=3 \nu_{0} / 10$ & 7 & 7 & 7 & 7 & 7 & 7 \\
\hline \multirow{3}{*}{$\mathbb{D}$} & \multirow{3}{*}{$1 / 10$} & $\nu_{1}=\nu_{0} / 10$ & 22 & 23 & 23 & 22 & 22 & 23 \\
& & $\nu_{1}=2 \nu_{0} / 10$ & 23 & 26 & 27 & 24 & 26 & 27 \\
& & $\nu_{1}=3 \nu_{0} / 10$ & 26 & 30 & 32 & 26 & 30 & 33 \\
\hline \multirow{3}{*}{$\mathbb{D}$} & \multirow{3}{*}{$1 / 50$} & $\nu_{1}=\nu_{0} / 10$ & 79 & 91 & 98 & 79 & 92 & 98 \\
& & $\nu_{1}=2 \nu_{0} / 10$ & 85 & 98 & 108 & 85 & 97 & 108 \\
& & $\nu_{1}=3 \nu_{0} / 10$ & 92 & 110 & 122 & 94 & 112 & 123 \\
\hline \hline \multirow{3}{*}{$\mathbb{F}_{0}$} & \multirow{3}{*}{1} & $\nu_{1}=\nu_{0} / 10$ & 5 & 6 & 6 & 6 & 6 & 6 \\
& & $\nu_{1}=2 \nu_{0} / 10$ & 7 & 9 & 9 & 7 & 8 & 9 \\
& & $\nu_{1}=3 \nu_{0} / 10$ & 9 & 11 & 12 & 9 & 11 & 12 \\
\hline \multirow{3}{*}{$\mathbb{F}_{0}$} & \multirow{3}{*}{$1 / 10$} & $\nu_{1}=\nu_{0} / 10$ & 6 & 7 & 7 & 6 & 7 & 7 \\
& & $\nu_{1}=2 \nu_{0} / 10$ & 9 & 10 & 10 & 9 & 10 & 10 \\
& & $\nu_{1}=3 \nu_{0} / 10$ & 12 & 14 & 14 & 12 & 14 & 14 \\
\hline \multirow{2}{*}{$\mathbb{F}_{0}$} & \multirow{3}{*}{$1 / 50$} & $\nu_{1}=\nu_{0} / 10$ & 7 & 7 & 8 & 7 & 8 & 8 \\
& & $\nu_{1}=2 \nu_{0} / 10$ & 11 & 12 & 13 & 11 & 12 & 13 \\
& & $\nu_{1}=3 \nu_{0} / 10$ & 16 & 19 & 21 & 16 & 20 & 21 \\
\hline
\end{tabular}

TABLE 5.1

Right-preconditioned GMRES iterations with preconditioners $\mathbb{P}_{F}=\mathbb{F}_{0}$ and $\mathbb{P}_{F}=\mathbb{D}$ for two consecutive levels of spatial grid refinement (coarser grid (left columns) and finer grid (right columns))

5.2. Approximating the Schur complement. Our strategy for approximating the Schur complement, $\mathbb{S}:=\mathbb{B F}_{\nu}^{-1} \mathbb{B}^{T}$, is to replace $\mathbb{F}$ by the approximation $\mathbb{F}_{0}$. That is,

$$
\begin{aligned}
\mathbb{S} & =\left[I \otimes B_{x}, I \otimes B_{y}\right] \mathbb{F}_{\nu}^{-1}\left[I \otimes B_{x}, I \otimes B_{y}\right]^{T} \\
& \approx\left[I \otimes B_{x}, I \otimes B_{y}\right]\left(I \otimes \boldsymbol{F}_{0}\right)^{-1}\left[I \otimes B_{x}, I \otimes B_{y}\right]^{T} \\
& =\left(I \otimes B_{x}\right)\left(I \otimes F_{0}^{-1}\right)\left(I \otimes B_{x}^{T}\right)+\left(I \otimes B_{y}\right)\left(I \otimes F_{0}^{-1}\right)\left(I \otimes B_{y}^{T}\right) \\
& =I \otimes\left(B \boldsymbol{F}_{0}^{-1} B^{T}\right)=: I \otimes S_{0}=: \mathbb{P}_{S} .
\end{aligned}
$$

The advantage of this is obvious. At each nonlinear iteration, the resulting approximation $\mathbb{P}_{S}$ is a block-diagonal version of the pressure Schur complement $S_{0}$ associated with the deterministic saddle-point matrix $(2.6)$ whose $(1,1)$ block is given by

$$
\boldsymbol{F}_{\nu}=\nu_{0} \boldsymbol{A}+\boldsymbol{N}\left(\mathbb{E}\left[\vec{u}_{h k}\right]\right) .
$$

Combining the convection-diffusion approximation (5.9) with the Schur complement approximation (5.12), we now have a specific exact preconditioner for (4.13),

$$
\mathscr{P}_{E}:=\left(\begin{array}{cc}
\mathbb{P}_{F} & \mathbb{B}^{T} \\
0 & -\mathbb{S}_{0}
\end{array}\right)=\left(\begin{array}{cc}
I \otimes \boldsymbol{F}_{0} & \mathbb{B}^{T} \\
0 & -I \otimes S_{0}
\end{array}\right) .
$$


Note that each solve with $\mathbb{P}_{F}$ requires $(k+1)$ solves with the deterministic matrix $\boldsymbol{F}_{0}$ and each solve with $\mathbb{S}_{0}$ involves $(k+1)$ solves with the deterministic Schur complement $S_{0}=B \boldsymbol{F}_{0}^{-1} B^{T}$. Although we don't anticipate to have to choose $k$ larger than say, six (see Section 4.2), this preconditioner is not practical as $S_{0}$ is dense. However, it does provide us with a starting point for evaluating performance, and we can immediately take advantage of existing deterministic algorithms to perform the solves with $S_{0}$ inexactly. Specifically, we apply the PCD and LSC approximations described in Section 2.2.

We follow the suggestion in [5] and implement PCD preconditioning (for $S_{0}$ ) via

$$
S_{0}^{-1}=\left(B \boldsymbol{F}_{0}^{-1} B^{T}\right)^{-1} \approx Q_{*}^{-1} F_{0}^{p} A_{*}^{-1} .
$$

$Q_{*}$ is the diagonal of the pressure mass matrix $Q_{p}$ and $A_{*}:=B \boldsymbol{M}_{*}^{-1} B^{T}$, where $\boldsymbol{M}_{*}$ is the diagonal of the velocity mass matrix $M$, with scalar components $M$, defined by

$$
\begin{aligned}
Q_{p} & :=\left[Q_{p}\right]_{i j}=\left(\psi_{i}, \psi_{j}\right), \quad i, j,=1, \ldots, n_{p}, \\
M & :=[M]_{i j}=\left(\phi_{i}, \phi_{j}\right), \quad i, j=1, \ldots, n_{u} .
\end{aligned}
$$

The key new ingredient is the $n_{p} \times n_{p}$ mean pressure convection-diffusion matrix

$$
F_{0}^{p}:=\left[F_{0}^{p}\right]_{i j}=\nu_{0}\left(\nabla \psi_{i}, \nabla \psi_{j}\right)+\left(\mathbb{E}\left[\vec{u}_{h k}\right] \cdot \nabla \psi_{i}, \nabla \psi_{j}\right), \quad i, j=1, \ldots, n_{p} .
$$

From deterministic studies, we know that the PCD approximation for $S_{0}$ is robust with respect to the discretization parameter $h$ but performance deteriorates slowly as the Reynolds number is increased. Replacing $S_{0}$ with the PCD approximation in (5.14) yields an alternative preconditioner which we denote by $\mathscr{P}_{P C D}$.

Similarly, we implement LSC preconditioning for $S_{0}$ via

$$
S_{0}^{-1}=\left(B \boldsymbol{F}_{0}^{-1} B^{T}\right)^{-1} \approx A_{*}^{-1}\left(B \boldsymbol{M}_{*}^{-1} \boldsymbol{F}_{0} \boldsymbol{M}_{*}^{-1} B^{T}\right) A_{*}^{-1},
$$

where $\boldsymbol{M}_{*}$ and $A_{*}$ are defined as for the PCD approximation. Using this approximation in (5.14) yields a third preconditioner, $\mathscr{P}_{L S C}$. From deterministic studies, we anticipate that the performance of this scheme will deteriorate slightly with mesh refinement (at least when $\nu_{0}=O(1)$ ) and for a fixed spatial refinement there will be a slight deterioration in performance as the Reynolds number is increased.

5.3. Initial saddle-point system. Before testing the preconditioners, we discuss the choice of initial guess for the Picard iteration. Each GMRES iteration, in each Picard step, requires a matrix-vector product with the coefficient matrix in (4.13). The cost of this rises from $O\left(n_{u}+n_{p}\right)$ (in the deterministic case) to $\left.O(k+1)^{2} n_{u}+k n_{p}\right)$. Although this is acceptable for small values of $k$, a smart choice of initial guess can reduce the number of Picard steps and thus the total number of matrix-vector products.

A natural starting point is the simpler symmetric saddle-point system,

$$
\left(\begin{array}{cc}
\left(\nu_{0} I+\nu_{1} G_{1}\right) \otimes \boldsymbol{A} & \mathbb{B}^{T} \\
\mathbb{B} & 0
\end{array}\right)\left(\begin{array}{l}
\boldsymbol{u}^{0} \\
\boldsymbol{p}^{0}
\end{array}\right)=\left(\begin{array}{l}
\boldsymbol{f}^{u} \\
\boldsymbol{f}^{p}
\end{array}\right)
$$

which corresponds to the stochastic Galerkin approximation, in $\boldsymbol{X}_{E}^{h} \otimes S^{k}$ and $M^{h} \otimes S^{k}$, for the linear Stokes problem (3.13)-(3.16). Once the discrete solution to (5.20) is obtained, we possess the coefficients needed to represent the initial approximations $\vec{u}_{h k}^{0}$ and $p_{h k}^{0}$ to the stochastic Galerkin Navier-Stokes solutions, in the same spaces, and we can proceed with the Picard iteration (4.7)-(4.8).

Observe that the Schur complement matrix associated with (5.20) is

$$
\mathbb{S}_{\text {stokes }}=\left(\nu_{0} I+\nu_{1} G_{1}\right)^{-1} \otimes B \boldsymbol{A}^{-1} B^{T}:=\left(\nu_{0} I+\nu_{1} G_{1}\right)^{-1} \otimes S_{\text {stokes }},
$$

where $S_{\text {stokes }}$ is the Schur complement matrix of the deterministic Stokes problem (with $\nu=1$ ). The pressure mass matrix $Q_{p}$ is an excellent approximation for $S_{\text {stokes }}$ (see 
e.g., [4, Ch.5]) and we can solve (5.20) efficiently using MINRES with the preconditioner,

$$
\mathscr{P}_{E, \text { stokes }}:=\left(\begin{array}{cc}
\left(\nu_{0} I+\nu_{1} G_{1}\right) \otimes \boldsymbol{A} & 0 \\
0 & \left(\nu_{0} I+\nu_{1} G_{1}\right)^{-1} \otimes Q_{p}
\end{array}\right) .
$$

Indeed, the next result guaranteees that preconditioned MINRES converges for (5.20), in a number of iterations that is independent of $h, k, \nu_{0}$ and $\nu_{1}$.

Lemma 5.4. For $Q_{2}-Q_{1}$ finite element spatial discretisations, the eigenvalues of the stochastic Galerkin Stokes matrix in (5.20), preconditioned by $\mathscr{P}_{E, \text { stokes }}$ in (5.21), lie in the bounded intervals $[-1,-a] \cup\{1\} \cup[1+a, 2]$, where

$$
a:=-\frac{1}{2}+\frac{1}{2} \sqrt{1+4 \gamma_{h}^{2}}
$$

and $\gamma_{h}>0$ is the $Q_{2}-Q_{1}$ Stokes inf-sup constant, which is independent of $h$.

Proof. See e.g., [4, p.293], and note that the eigenvalues of the preconditioned stochastic Schur complement matrix $\left(\left(\nu_{0} I+\nu_{1} G_{1}\right) \otimes Q_{p}^{-1}\right) \mathbb{S}_{\text {stokes }}$ coincide with those of $Q_{p}^{-1} S_{\text {stokes }}$ (the preconditioned deterministic Schur complement matrix).

Following the discussion in Section 3.2, the stochastic Stokes velocity is

$$
\vec{u}(\vec{x}, \lambda)=\vec{u}_{d e t}(\vec{x}) \varphi_{0}(\lambda)=: \vec{u}_{0}(\vec{x}) \varphi_{0}(\lambda),
$$

where $\vec{u}_{\text {det }}$ is the deterministic Stokes velocity solution. In addition, $\nu(\lambda)=\nu_{0}+\nu_{1} \lambda=$ $\nu_{0} \varphi_{0}(\lambda)+\nu_{1} \varphi_{1}(\lambda)$ for the choice $(3.9)$, and since $p(\vec{x}, \lambda)=\nu(\lambda) p_{\text {det }}(\vec{x})$,

$$
p(\vec{x}, \lambda)=\nu_{0} p_{\text {det }}(\vec{x}) \varphi_{0}(\lambda)+\nu_{1} p_{\text {det }}(\vec{x}) \varphi_{1}(\lambda)=: p_{0}(\vec{x}) \varphi_{0}(\lambda)+p_{1}(\vec{x}) \varphi_{1}(\lambda) .
$$

The exact Stokes solution has linear dependence on the image coordinate $\lambda$. There is no need then to approximate it with a stochastic Galerkin method with $k>1$. Otherwise, the discrete solution of (5.20) has redundant zero blocks, which approximate the zero PC coefficients of the polynomials $\psi_{2}, \ldots, \psi_{k}$. That is,

$$
\boldsymbol{u}^{0}=\left(\begin{array}{c}
\boldsymbol{u}_{0}^{0} \\
\boldsymbol{o} \\
\boldsymbol{o} \\
\vdots \\
\boldsymbol{o}
\end{array}\right), \quad \boldsymbol{p}^{0}=\left(\begin{array}{c}
\boldsymbol{p}_{0}^{0} \\
\boldsymbol{p}_{1}^{0} \\
\boldsymbol{0} \\
\vdots \\
\boldsymbol{o}
\end{array}\right),
$$

(in exact arithmetic). We can therefore construct the initial guess for the Navier-Stokes problem by solving the smaller stochastic Galerkin Stokes system (5.20) corresponding to the polynomial space $S^{1}$. This yields discrete components $\boldsymbol{u}_{0}^{0}, \boldsymbol{u}_{0}^{1} \approx \boldsymbol{o}, \boldsymbol{p}_{0}^{0}$, and $\boldsymbol{p}_{1}^{0}$, which can then be used to populate the non-zero blocks of $\boldsymbol{u}^{0}$ and $\boldsymbol{p}^{0}$ in (5.25).

If the Stokes solution is used as the initial guess, the initial velocity is deterministic and the convection-diffusion matrix at the first Picard step is simply,

$$
\mathbb{F}_{\nu}^{1}:=\left(\nu_{0} I+\nu_{1} G_{1}\right) \otimes \boldsymbol{A}+H_{0} \otimes \boldsymbol{N}_{0}=I \otimes\left(\nu_{0} \boldsymbol{A}+\boldsymbol{N}_{0}\right)+\nu_{1} G_{1} \otimes \boldsymbol{A} .
$$

This resembles a block-diagonal version of the deterministic Navier-Stokes convectiondiffusion matrix, with viscosity $\nu_{0}$ and convection coefficient given by the Stokes velocity.

6. Numerical results. We applied the exact preconditioner $\mathscr{P}_{E}$ from Section 5.2, and the practical variants $\mathscr{P}_{P C D}$ and $\mathscr{P}_{L S C}$, to the saddle-point systems arising in the Picard iteration for the stochastic Galerkin discretisation of the backward-facing step problem. Below, we report the number of preconditioned GMRES iterations required to solve the saddle-point system corresponding to the final Picard step, for a range of discretisation and statistical parameters. The stopping tolerance for the residual error in both the nonlinear iteration, and the inner GMRES iterations, was set to $10^{-6}$. The 
total number of Picard steps required depends on the problem parameters, which we vary, but is less than twenty-five in all the reported experiments. The initial guess for the nonlinear iteration was obtained by solving the Stokes problem, as discussed in Section 5.3, using MINRES with the preconditioner (5.21).

We report results for two different values of $\nu_{0}$. Recall that when $\nu_{0}$ decreases, $\mathbb{E}[\operatorname{Re}]$ increases (see Table 3.1). To accurately capture the dynamics of the flow we need to increase the channel length, $L$ (i.e., change the spatial domain). For each choice of $\nu_{0}$ we consider two successively refined spatial grids. The stretch factor was set to 1.2 and the dimensions of the resulting finite element spaces are summarized in Table 6.1. For $\nu_{0}=1 / 50, L=5$ suffices and in that case, the fine grid is the same as in Figure 2.3.

\begin{tabular}{r|rr|rr}
\hline & \multicolumn{2}{|c|}{ Coarse grid } & \multicolumn{2}{c}{ Fine grid } \\
\hline$\nu_{0}$ & $1 / 50$ & $1 / 100$ & $1 / 50$ & $1 / 100$ \\
$L$ & 5 & 10 & 5 & 10 \\
\hline$n_{u}$ & 2,183 & 3,803 & 6,321 & 10,221 \\
$n_{p}$ & 565 & 990 & 1,625 & 2,615 \\
$2 n_{u}+n_{p}$ & 4,871 & 8,596 & 14,267 & 23,057 \\
\hline \multicolumn{4}{c}{ TABLE 6.1}
\end{tabular}

Number of deterministic degrees of freedom associated with two successive refinements of the $Q_{2}-$ $Q_{1}$ finite element mesh and varying length of channel domain, $L$.

The number of MINRES iterations required to solve the system (5.20) for the initial guess was observed to be independent of all the problem parameters (see Table 6.2). Note that for a fixed spatial domain, the iteration counts are actually independent of $\nu_{0}$ and $\nu_{1}$. However, when we decrease $\nu_{0}$, we increase $L$, and the inf-sup constant appearing in the eigenvalue bounds in Lemma 5.4 decreases. This explains the slight rise in iterations as $L$ increases from five (when $\nu_{0}=1 / 50$ ) to ten (when $\nu_{0}=1 / 100$ ).

\begin{tabular}{r|cc|cc}
\hline & \multicolumn{2}{|c|}{ Coarse grid } & \multicolumn{2}{c}{ Fine grid } \\
$\nu_{0}$ & $1 / 50$ & $1 / 100$ & $1 / 50$ & $1 / 100$ \\
\hline$\nu_{1}=\nu_{0} / 10$ & 42 & 50 & 42 & 50 \\
$\nu_{1}=2 \nu_{0} / 10$ & 42 & 50 & 42 & 50 \\
$\nu_{1}=3 \nu_{0} / 10$ & 42 & 50 & 42 & 50 \\
\hline \multicolumn{5}{|c}{ TABLE 6.2}
\end{tabular}

Preconditioned MINRES iterations for the initial Stokes system (with $k=1$ ).

GMRES iteration counts for the Picard systems are recorded in Tables 6.3 and 6.4 and convergence curves for the relative residual errors, in the case $\nu_{0}=1 / 50$, are shown in Figure 6.1. As anticipated, the exact preconditioner $\mathscr{P}_{E}$ is perfectly robust: both with respect to the spatial approximation and with respect to the degree of spectral approximation, $k$. Recall that all versions of the preconditioner are based on the mean convection-diffusion approximation $\mathbb{P}_{F}=\mathbb{F}_{0}$ and we know (from Lemma 5.3 and Table $5.1)$ that the quality of this approximation deteriorates slightly as $\nu_{1} / \nu_{0}$ increases. This is of course reflected in the GMRES iteration counts for the saddle-point problems.

The LSC preconditioning results are less encouraging and the deterioration in the convergence rate is apparent when the spatial grid is refined. This is not a surprise as the grid dependence of LSC is known from the deterministic case. In contrast, the PCD preconditioner results are very promising - the convergence rate stays within a factor of two or three of the exact preconditioner $\mathscr{P}_{E}$ and the approximation is perfectly robust with respect to the discretisation parameters $h$ and $k$. For the range of Reynolds numbers considered, the dependence on the ratio $\nu_{1} / \nu_{0}$ is also acceptable. 


\begin{tabular}{l|c|c|ccc|ccc}
\hline & & & \multicolumn{3}{|c|}{ Coarse grid } & \multicolumn{4}{c}{ Fine grid } \\
& & $\mathbb{E}[\mathrm{Re}]$ & $k=2$ & 4 & 6 & $k=2$ & 4 & 6 \\
\hline \multirow{3}{*}{$\mathscr{P}_{E}$} & $\nu_{1}=\nu_{0} / 10$ & 67 & 14 & 14 & 14 & 14 & 14 & 15 \\
& $\nu_{1}=2 \nu_{0} / 10$ & 70 & 18 & 20 & 21 & 14 & 20 & 21 \\
& $\nu_{1}=3 \nu_{0} / 10$ & 74 & 25 & 28 & 29 & 25 & 28 & 29 \\
\hline \multirow{3}{*}{$\mathscr{P}_{P C D}$} & $\nu_{1}=\nu_{0} / 10$ & 67 & 37 & 38 & 39 & 37 & 39 & 39 \\
& $\nu_{1}=2 \nu_{0} / 10$ & 70 & 43 & 44 & 50 & 44 & 48 & 50 \\
& $\nu_{1}=3 \nu_{0} / 10$ & 74 & 53 & 56 & 61 & 54 & 58 & 62 \\
\hline \multirow{3}{*}{$\mathscr{P}_{L S C}$} & $\nu_{1}=\nu_{0} / 10$ & 67 & 25 & 26 & 27 & 43 & 49 & 52 \\
& $\nu_{1}=2 \nu_{0} / 10$ & 70 & 31 & 34 & 36 & 48 & 58 & 63 \\
& $\nu_{1}=3 \nu_{0} / 10$ & 74 & 35 & 45 & 48 & 51 & 68 & 77 \\
\hline
\end{tabular}

Right-preconditioned GMRES iterations for the final Picard system for the case $\nu_{0}=1 / 50, L=5$.

\begin{tabular}{l|c|c|ccc|ccc}
\hline & & & \multicolumn{3}{|c|}{ Coarse grid } & \multicolumn{4}{c}{ Fine grid } \\
& & $\mathbb{E}[\mathrm{Re}]$ & $k=2$ & 4 & 6 & $k=2$ & 4 & 6 \\
\hline \multirow{3}{*}{$\mathscr{P}_{E}$} & $\nu_{1}=\nu_{0} / 10$ & 135 & 16 & 16 & 17 & 16 & 17 & 17 \\
& $\nu_{1}=2 \nu_{0} / 10$ & 139 & 22 & 23 & 24 & 22 & 24 & 24 \\
& $\nu_{1}=3 \nu_{0} / 10$ & 148 & 29 & 36 & 38 & 29 & 36 & 37 \\
\hline \multirow{3}{*}{$\mathscr{P}_{P C D}$} & $\nu_{1}=\nu_{0} / 10$ & 135 & 44 & 46 & 48 & 44 & 47 & 48 \\
& $\nu_{1}=2 \nu_{0} / 10$ & 139 & 53 & 59 & 64 & 51 & 58 & 64 \\
& $\nu_{1}=3 \nu_{0} / 10$ & 148 & 73 & 87 & 97 & 74 & 81 & 84 \\
\hline \multirow{3}{*}{$\mathscr{P}_{L S C}$} & $\nu_{1}=\nu_{0} / 10$ & 135 & 44 & 46 & 51 & 62 & 74 & 81 \\
& $\nu_{1}=2 \nu_{0} / 10$ & 139 & 55 & 60 & 63 & 70 & 83 & 92 \\
& $\nu_{1}=3 \nu_{0} / 10$ & 148 & 67 & 78 & 83 & 79 & 97 & 109 \\
\hline
\end{tabular}

Right-preconditioned GMRES iterations for the final Picard system for the case $\nu_{0}=1 / 100, L=10$.
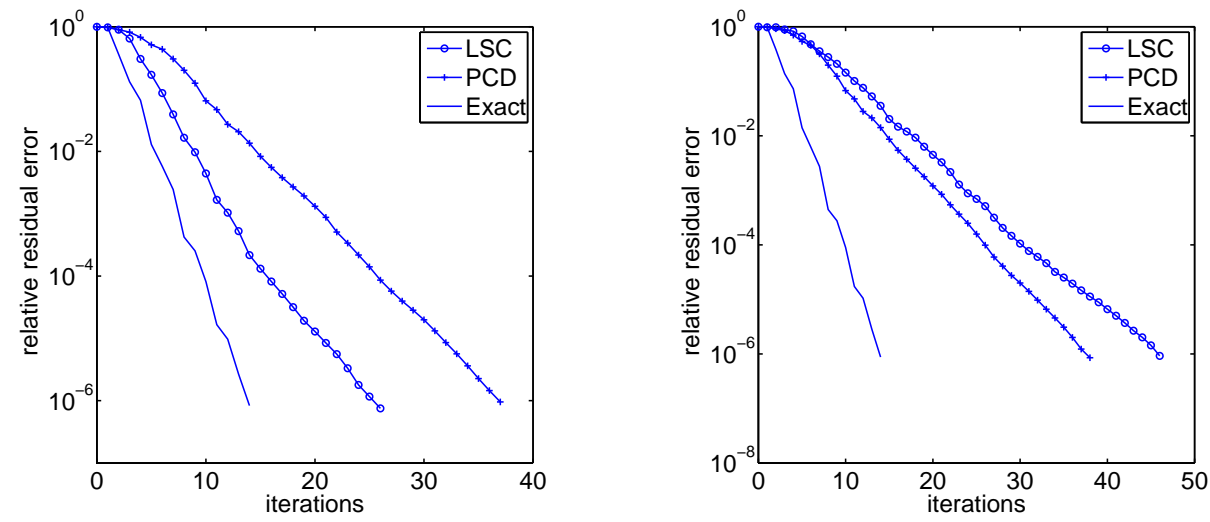

FIG. 6.1. GMRES convergence when solving the discretized stochastic flow problem (4.13) for $\nu_{0}=1 / 50, \nu_{1}=1 / 500$ and $k=3$, on a coarse grid (left) and a fine grid (right), with $L=5$.

7. Summary. In this study we have developed a mean-based preconditioner for linear algebra systems that arise from a stochastic Galerkin mixed formulation of the nonlinear steady-state Navier-Stokes equations with stochastically linear random data. If stochastic Galerkin methods are to be competitive with traditional deterministic methodologies based on sampling techniques, then we need fast and robust preconditioning techniques to solve the large linear algebra systems that arise. The strategy that is developed herein builds on state-of-the-art preconditioners developed for deter- 
ministic flow problems - so we can immediately implement it using existing software. Computational results confirm that our strategy gives an effective way of solving the coupled systems that arise, especially when the fluctuations in the data are not too large relative to their mean values. We intend to look at more complex unsteady flow problems, as well as extending our methodology to cover stochastically nonlinear data

models in the future.

\section{REFERENCES}

[1] Jonathan Boyle, Milan Mihajlović and Jennifer Scott, HSL_MI20: an efficient AMG preconditioner for finite element problems in 3D, Internat. J. Numer. Methods Engrg, 82 (2010), $64-98$.

[2] Howard C. Elman, Alison Ramage and David J. Silvester, Algorithm 866: IFISS, a MATLAB toolbox for modelling incompressible flow, ACM Trans. Math. Softw., 33, (2007), 2-14.

[3] Howard C. Elman, David J. Silvester and Andrew J. Wathen, Performance and analysis of saddle point preconditioners for the discrete steady-state Navier-Stokes equations, Numer. Math., 90 (2002), 665-688.

[4] Howard C. Elman, David J. Silvester and Andrew J. Wathen, "Finite Elements and Fast Iterative Solvers: With Applications in Incompressible Fluid Dynamics," Oxford University Press, New York, 2005. xiv+400 pp. ISBN: 978-0-19-852868-5; 0-19-852868-X.

[5] Howard C. Elman and Ray S. Tuminaro, Boundary conditions in approximate commutator preconditioners for the Navier-Stokes equations, Electron. Trans. Numer. Anal., 35 (2009), $257-280$.

[6] Oliver G. Ernst and Elisabeth Ullmann, Stochastic Galerkin matrices, SIAM J. Sci. Comput., 31 (2009), 1424-1447.

[7] Oliver G. Ernst, Catherine E. Powell, David J. Silvester and Elisabeth Ullmann, Efficient solvers for a linear stochastic Galerkin mixed formulation of diffusion problems with random data, SIAM J. Matrix. Anal. Appl., 31 (2010), 1848-1872.

[8] P.M. Gresho, D.K. Gartling, J.R. Torczynski, K.A. Cliffe, K.H. Winters, T.J. Garratt, A. Spence and J.W. Goodrich, Is the steady viscous incompressible two-dimensional flow over a backward-facing step at Re=800 stable?, Internat. J. Numer. Methods Fluids, 17 (1993), 501-541.

[9] David Kay, Daniel Loghin and Andrew Wathen, A preconditioner for the steady-state NavierStokes equations, SIAM J. Sci. Comput., 24 (2002), 237-256.

[10] O. P. Le Maître and O. M. Knio, "Spectral Methods for Uncertainty Quantification: With Applications to Computational Fluid Dynamics," Springer, New York, 2010. xvi+536 pp. ISBN: 978-90-481-3519-6.

[11] Catherine E. Powell and Howard C. Elman, Block-diagonal preconditioning for spectral stochastic finite-element systems, IMA J. Numer. Anal., 29 (2009), 350-375.

[12] David Silvester, Howard Elman, David Kay and Andrew Wathen, Efficient preconditioning of the linearised Navier-Stokes equations for incompressible flow, J. Comput. Appl. Math., 128 (2001), 261-279.

[13] David Silvester, Howard Elman and Alison Ramage, "Incompressible Flow and Iterative Solver Software (IFISS)," Version 3.2, 2012. Available from http://www.manchester.ac.uk/ifiss/.

[14] Elisabeth Ullmann, Howard C. Elman and Oliver G. Ernst, Efficient iterative solvers for stochastic Galerkin discretizations of log-transformed random diffusion problems, UMIACS-TR-201104, (2011).

[15] Dongbin Xiu, "Numerical Methods for Stochastic Computations: A spectral method approach," Princeton University Press, Princeton, NJ, 2010. xiv+127 pp. ISBN: 978-0-691-14212-8. 